\title{
A new approach to the 2-regularity of the $\ell$-abelian complexity of 2 -automatic sequences
}

\author{
Aline Parreau* \\ LIRIS \\ University of Lyon, CNRS \\ Lyon, France \\ aline.parreau@univ-lyon1.fr \\ Eric Rowland ${ }^{\dagger}$ \\ Department of Mathematics \\ University of Liege \\ Liege, Belgium \\ erowland@ulg.ac.be
}

\author{
Michel Rigo \\ Department of Mathematics \\ University of Liege \\ Liege, Belgium \\ M.Rigo@ulg.ac.be \\ Élise Vandomme \\ Department of Mathematics \\ University of Liege \\ Liege, Belgium \\ and \\ Institut Fourier \\ University of Grenoble \\ Grenoble, France \\ E.Vandomme@ulg.ac.be
}

Submitted: Jun 20, 2014; Accepted: Jan 17, 2015; Published: Feb 9, 2015

Mathematics Subject Classifications: 68R15, 11B85, 05A05

\begin{abstract}
We prove that a sequence satisfying a certain symmetry property is 2-regular in the sense of Allouche and Shallit, i.e., the $\mathbb{Z}$-module generated by its 2-kernel is finitely generated. We apply this theorem to develop a general approach for studying the $\ell$-abelian complexity of 2 -automatic sequences. In particular, we prove that the period-doubling word and the Thue-Morse word have 2-abelian complexity sequences that are 2-regular. Along the way, we also prove that the 2-block codings of these two words have 1-abelian complexity sequences that are 2-regular.
\end{abstract}

*This work has been done when this author was an FNRS post-doctoral fellow at the University of Liege.

${ }^{\dagger}$ BeIPD-COFUND post-doctoral fellow at the University of Liege. 


\section{Contents}

1 Introduction 2

$\begin{array}{lll}2 & \text { Sequences satisfying a reflection symmetry } & 7\end{array}$

3 About regular sequences and words $\quad 11$

4 Abelian complexity of $\operatorname{block}(\mathrm{p}, 2) \quad 13$

4.1 Proof of Proposition $20 \ldots \ldots \ldots \ldots$. . . . . . . . . . . . . 14

$4.2 \Delta_{0}(n)_{n \geqslant 0}$ is 2-regular, $\left(\min _{0}(n) \bmod 2\right)_{n \geqslant 0}$ is 2 -automatic . . . . . . . 16

4.3 Another proof of the 2-regularity of $\mathcal{P}_{\mathbf{x}}^{(1)}(n)_{n \geqslant 0} \ldots \ldots \ldots \ldots . \ldots . \ldots . \ldots 22$

$5 \quad 2$-abelian complexity of the period-doubling word 23

6 Abelian complexity of $\operatorname{block}(\mathrm{t}, 2) \quad 27$

6.1 Proof of Proposition $43 \ldots \ldots \ldots \ldots \ldots$

$6.2 \Delta_{12}(n)_{n \geqslant 0}$ is 2-regular, $\left(\min _{12}(n) \bmod 2\right)_{n \geqslant 0}$ is 2 -automatic . . . . . . . . 32

$\begin{array}{lll}7 & 2 \text {-abelian complexity of the Thue-Morse word } & 38\end{array}$

8 Conclusions $\quad 42$

\section{Introduction}

This paper is about some structural properties of integer sequences that occur naturally in combinatorics on words. Since the fundamental work of Cobham [8], the so-called automatic sequences have been extensively studied. We refer the reader to [3] for basic definitions and properties. These infinite words over a finite alphabet can be obtained by iterating a prolongable morphism of constant length to get an infinite word (and then, an extra letter-to-letter morphism, also called coding, may be applied). As a fundamental example, the Thue-Morse word $\mathbf{t}=\sigma^{\omega}(0)=0110100110010110 \cdots$ is a fixed point of the morphism $\sigma$ over the free monoid $\{0,1\}^{*}$ defined by $\sigma(0)=01, \sigma(1)=10$. Similarly, the period-doubling word $\mathbf{p}=\psi^{\omega}(0)=01000101010001000100 \cdots$ is a fixed point of the morphism $\psi$ over $\{0,1\}^{*}$ defined by $\psi(0)=01, \psi(1)=00$. We will discuss again these two examples of 2-automatic sequences.

Since an infinite word is just a sequence over $\mathbb{N}$ taking values in a finite alphabet, we use the terms 'infinite word' and 'sequence' interchangeably.

Let $k \geqslant 2$ be an integer. One characterization of $k$-automatic sequences is that their $k$-kernels are finite; see [9] or [3, Section 6.6].

Definition 1. The $k$-kernel of a sequence $\mathbf{s}=s(n)_{n \geqslant 0}$ is the set

$$
\mathcal{K}_{k}(\mathbf{s})=\left\{s\left(k^{i} n+j\right)_{n \geqslant 0}: i \geqslant 0 \text { and } 0 \leqslant j<k^{i}\right\} .
$$


For instance, the 2-kernel $\mathcal{K}_{2}(\mathbf{t})$ of the Thue-Morse word contains exactly two elements, namely $\mathbf{t}$ and $\sigma^{\omega}(1)$.

A natural generalization of automatic sequences to sequences on an infinite alphabet is given by the notion of $k$-regular sequences. We will restrict ourselves to sequences taking integer values only.

Definition 2. Let $k \geqslant 2$ be an integer. A sequence $\mathbf{s}=s(n)_{n \geqslant 0} \in \mathbb{Z}^{\mathbb{N}}$ is $k$-regular if $\left\langle\mathcal{K}_{k}(\mathbf{s})\right\rangle$ is a finitely-generated $\mathbb{Z}$-module, i.e., there exist a finite number of sequences $t_{1}(n)_{n \geqslant 0}, \ldots, t_{\ell}(n)_{n \geqslant 0}$ such that every sequence in the $k$-kernel $\mathcal{K}_{k}(\mathbf{s})$ is a $\mathbb{Z}$-linear combination of the $t_{r}$ 's. Otherwise stated, for all $i \geqslant 0$ and for all $j \in\left\{0, \ldots, k^{i}-1\right\}$, there exist integers $c_{1}, \ldots, c_{\ell}$ such that

$$
\forall n \geqslant 0, \quad s\left(k^{i} n+j\right)=\sum_{r=1}^{\ell} c_{r} t_{r}(n) .
$$

There are many natural examples of $k$-regular sequences $[1,2]$. There is a convenient matrix representation for $k$-regular sequences which leads to an efficient algorithm for computing the values of such a sequence (and many related quantities). See also [4, Chapter 5] for connections with rational series. In particular, a sequence taking finitely many values is $k$-regular if and only if it is $k$-automatic. The $k$-regularity of a sequence provides us with structural information about how the different terms are related to each other.

A classical measure of complexity of an infinite word $\mathbf{x}$ is its factor complexity $\mathcal{P}_{\mathbf{x}}^{(\infty)}$ : $\mathbb{N} \rightarrow \mathbb{N}$ which maps $n$ to the number of distinct factors of length $n$ occurring in $\mathbf{x}$. It is well known that a $k$-automatic sequence $\mathbf{x}$ has a $k$-regular factor complexity function and the sequence $\left(\mathcal{P}_{\mathbf{x}}^{(\infty)}(n+1)-\mathcal{P}_{\mathbf{x}}^{(\infty)}(n)\right)_{n \geqslant 0}$ is $k$-automatic. See [6, 7] for a proof and relevant extensions. As an example, again for the Thue-Morse word, we have

$$
\mathcal{P}_{\mathbf{t}}^{(\infty)}(2 n+1)=2 \mathcal{P}_{\mathbf{t}}^{(\infty)}(n+1) \text { and } \mathcal{P}_{\mathbf{t}}^{(\infty)}(2 n)=\mathcal{P}_{\mathbf{t}}^{(\infty)}(n+1)+\mathcal{P}_{\mathbf{t}}^{(\infty)}(n)
$$

for all $n \geqslant 2$. See also [10] where a formula was obtained for the factor complexity of fixed points of some uniform morphisms.

Recently there has been a renewal of interest in abelian notions arising in combinatorics on words (e.g., avoiding abelian or $\ell$-abelian patterns, abelian bordered words, etc.). For instance, two finite words $u$ and $v$ are abelian equivalent if one is obtained by permuting the letters of the other one, i.e., the two words share the same Parikh vector, $\Psi(u)=\Psi(v)$. Since the Thue-Morse word is an infinite concatenation of factors 01 and 10, this word is abelian periodic of period 2. The abelian complexity of an infinite word $\mathbf{x}$ is a function $\mathcal{P}_{\mathbf{x}}^{(1)}: \mathbb{N} \rightarrow \mathbb{N}$ which maps $n$ to the number of distinct factors of length $n$ occurring in $\mathbf{x}$, counted up to abelian equivalence. Madill and Rampersad [15] provided the first example of regularity in this setting: the abelian complexity of the paper-folding word (which is another typical example of an automatic sequence) is unbounded and 2-regular.

Let $\ell \geqslant 1$ be an integer. Based on [12] the notions of abelian equivalence and thus abelian complexity were recently extended to $\ell$-abelian equivalence and $\ell$-abelian complexity [13]. 
Definition 3. Let $u, v$ be two finite words. We let $|u|_{v}$ denote the number of occurrences of the factor $v$ in $u$. Two finite words $x$ and $y$ are $\ell$-abelian equivalent if $|x|_{v}=|y|_{v}$ for all words $v$ of length $|v| \leqslant \ell$.

As an example, the words 011010011 and 001101101 are 2-abelian equivalent but not 3-abelian equivalent (the factor 010 occurs in the first word but not in the second one). Hence one can define the function $\mathcal{P}_{\mathbf{x}}^{(\ell)}: \mathbb{N} \rightarrow \mathbb{N}$ which maps $n$ to the number of distinct factors of length $n$ occurring in the infinite word $\mathbf{x}$, counted up to $\ell$-abelian equivalence. That is, we count $\ell$-abelian equivalence classes partitioning the set of factors $\operatorname{Fac}_{\mathbf{x}}(n)$ of length $n$ occurring in $\mathbf{x}$. In particular, for any infinite word $\mathbf{x}$, we have for all $n \geqslant 0$

$$
\mathcal{P}_{\mathbf{x}}^{(1)}(n) \leqslant \cdots \leqslant \mathcal{P}_{\mathbf{x}}^{(\ell)}(n) \leqslant \mathcal{P}_{\mathbf{x}}^{(\ell+1)}(n) \leqslant \cdots \leqslant \mathcal{P}_{\mathbf{x}}^{(\infty)}(n) .
$$

In this paper, we show that both the period-doubling word and the Thue-Morse word have 2-abelian complexity sequences which are 2-regular. The computations and arguments leading to these results permit us to exhibit some similarities between the two cases and a quite general scheme that we hope can be used again to prove additional regularity results. Indeed, one conjectures that any $k$-automatic sequence has an $\ell$-abelian complexity function that is $k$-regular.

We mention some other papers containing related work. In [14], the authors studied the asymptotic behavior of $\mathcal{P}_{\mathbf{t}}^{(\ell)}(n)$ and also derived some recurrence relations ${ }^{1}$ showing that the abelian complexity $\mathcal{P}_{\mathbf{p}}^{(1)}(n)_{n \geqslant 0}$ of the period-doubling word $\mathbf{p}$ is 2-regular. In [5], the abelian complexity of the fixed point $\mathbf{v}$ of the non-uniform morphism $0 \mapsto 012,1 \mapsto$ $02,2 \mapsto 1$ is studied and the authors obtain results similar to those discussed in this paper. Even though the authors of [5] are not directly interested in the $k$-regularity of $\mathcal{P}_{\mathbf{v}}^{(1)}(n)_{n \geqslant 0}$, they derive recurrence relations. From these relations, following the approach described in this paper, one can possibly prove some regularity result. In particular, the result of replacing in $\mathbf{v}$ all 2's by 0's leads back to the period-doubling word. Hence, Blanchet-Sadri et al. also proved some other relations about the abelian complexity of $\mathbf{p}$.

Given the first few terms of a sequence, one can easily conjecture the potential $k$ regularity of this sequence by exhibiting relations that should be satisfied; see [2, Section 6] for such a "predictive" algorithm that recognizes regularity. Of course, in such an algorithm, a finite examination does not lead to a proof of the $k$-regularity of a sequence. The first few terms of the 2-abelian complexity $\mathcal{P}_{\mathbf{t}}^{(2)}(n)_{n \geqslant 0}$ of the Thue-Morse word are

$$
1,2,4,6,8,6,8,10,8,6,8,8,10,10,10,8,8,6,8,10,10,8,10,12,12,10,12,12, \ldots
$$

The second and last authors of this paper conjectured the 2-regularity of the sequence $\mathcal{P}_{\mathbf{t}}^{(2)}(n)_{n \geqslant 0}$ (and proved some recurrence relations for this sequence) [17]. Recently, after hearing a talk given by the last author during the Representing Streams II meeting in January 2014, Greinecker proved the recurrence relations needed to prove the 2-regularity

\footnotetext{
${ }^{1}$ It seems that there is some subtle error in the relation for $\mathcal{P}_{\mathbf{p}}^{(1)}(4 n+2)$ proposed in [14, Lemma 6]. Correct relations are given by [5, Proposition 2] and could also be obtained by Theorem 4 and Proposition 47.
} 
of this sequence [11]. Hopefully, the two approaches are complementary: in this paper, we prove 2-regularity without exhibiting the explicit recurrence relations.

Let us now describe the content and organization of this paper.

In Section 2 we prove Theorem 4, which establishes the 2-regularity of a large family of sequences satisfying a recurrence relation with a parameter $c$ and $2^{\ell_{0}}$ initial conditions. The form of the recurrence implies that sequences in this family exhibit a reflection symmetry in the values taken over each interval $\left[2^{\ell}, 2^{\ell+1}\right)$ for $\ell \geqslant \ell_{0}$. For the special case of the Thue-Morse word, a similar property is shown in [11]. Computer experiments suggest that many 2-abelian complexity functions satisfy such a reflection property.

Theorem 4. Let $\ell_{0} \geqslant 0$ and $c \in \mathbb{Z}$. Suppose $s(n)_{n \geqslant 0}$ is a sequence such that, for all $\ell \geqslant \ell_{0}$ and for all $r$ such that $0 \leqslant r \leqslant 2^{\ell}-1$, we have

$$
s\left(2^{\ell}+r\right)= \begin{cases}s(r)+c & \text { if } r \leqslant 2^{\ell-1} \\ s\left(2^{\ell+1}-r\right) & \text { if } r>2^{\ell-1}\end{cases}
$$

Then $s(n)_{n \geqslant 0}$ is 2-regular.

The recurrence satisfied by $s(n)$ in Theorem 4 reads words from left to right, i.e., starting with the most significant digit. Our proof of this theorem will express sequences in the 2-kernel of $s(n)_{n \geqslant 0}$ as in Definition 2, starting with the least significant digit.

From Equation (1) one can get some information about the asymptotic behavior of the sequence $s(n)_{n \geqslant 0}$. We have $s(n)=O(\log n)$, and moreover

$$
s\left(\frac{4^{\ell+1}-1}{3}\right)=s\left(4^{\ell}+\cdots+4^{1}+4^{0}\right)=\left(\ell-\left\lfloor\frac{\ell_{0}-1}{2}\right\rfloor\right) c+s\left(\frac{4^{\left\lfloor\left(\ell_{0}+1\right) / 2\right\rfloor}-1}{3}\right)
$$

for $\ell \geqslant\left\lfloor\frac{\ell_{0}-1}{2}\right\rfloor$. At the same time, there are many subsequences of $s(n)_{n \geqslant 0}$ which are constant; for example, $s\left(2^{\ell}\right)=c$ for $\ell \geqslant \ell_{0}$.

Example 5. As an illustration of the reflection property described in Theorem 4, we consider the abelian complexity of the 2-block coding of the period-doubling word $\mathbf{p}$. (The recurrence satisfied by this sequence is given in Theorem 21.) Some values of this sequence are depicted in Figures 1 and 2.

In Section 3, we collect some general results and definitions about words and $k$-regular sequences (in particular stability properties of the set of $k$-regular sequences under sum and product) that are needed in the other parts of this paper.

In Section 4, we study the abelian complexity of the 2-block coding $\mathbf{x}=\operatorname{block}(\mathbf{p}, 2)$ of the period-doubling word $\mathbf{p}$. In particular, we consider the difference $\Delta_{0}(n)$ between the maximal and minimal numbers of 0's occurring in factors of length $n$ in block $(\mathbf{p}, 2)$. We prove that the sequences $\Delta_{0}(n)_{n \geqslant 0}$ and $\mathcal{P}_{\mathbf{x}}^{(1)}(n)_{n \geqslant 0}$ are 2-regular. In Section 5 , we study the 2-abelian complexity of $\mathbf{p}$. We show that the 2-regularity of $\Delta_{0}(n)_{n \geqslant 0}$ and $\mathcal{P}_{\mathbf{x}}^{(1)}(n)_{n \geqslant 0}$ implies the 2-regularity of $\mathcal{P}_{\mathbf{p}}^{(2)}(n)$. 

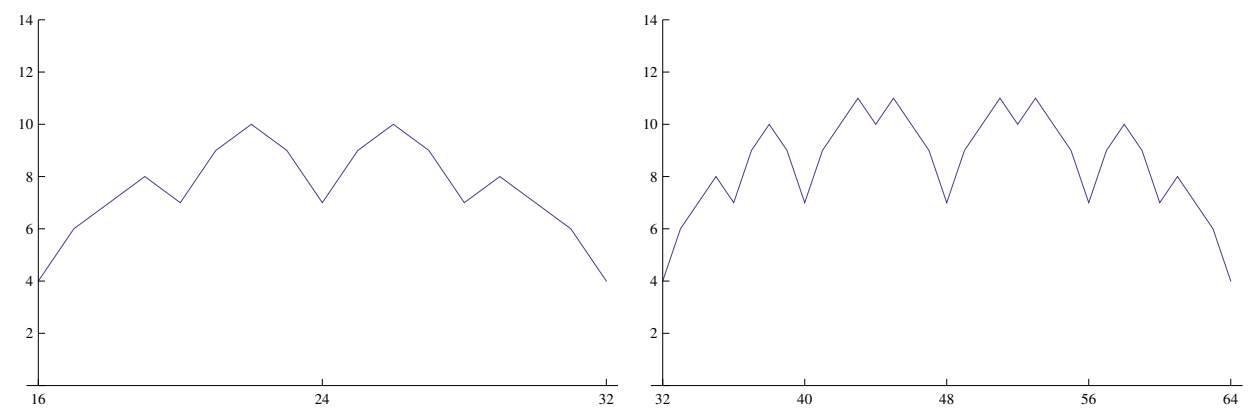

Figure 1: The abelian complexity of $\operatorname{block}(\mathbf{p}, 2)$ on the intervals $[16,32]$ and $[32,64]$.

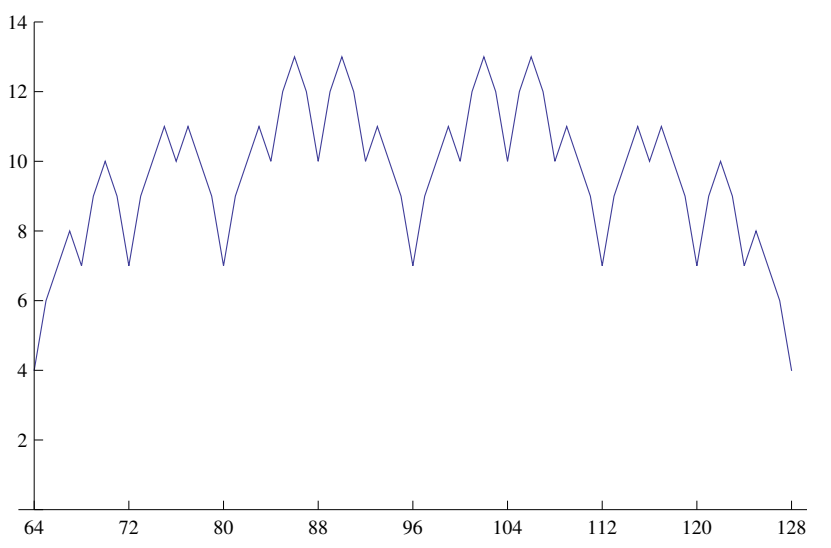

Figure 2: The abelian complexity of $\operatorname{block}(\mathbf{p}, 2)$ on the interval $[64,128]$. 
Sections 6 and 7 share some similarities with Sections 4 and 5 . The reader will see that the strategy used to prove the 2-regularity of $\mathcal{P}_{\mathbf{p}}^{(2)}(n)$ can also be applied to the Thue-Morse word. Nevertheless, some differences do not permit us to treat the two cases within a completely unified framework.

In Section 6, we study the abelian complexity of the 2-block coding $\mathbf{y}=\operatorname{block}(\mathbf{t}, 2)$ of the Thue-Morse word $\mathbf{t}$. We define $\Delta_{12}(n)$ to be the difference between the maximal total and minimal total numbers of 1's and 2's occurring in factors of length $n$ in block(t, 2). It turns out that $\Delta_{12}(n)+1=\mathcal{P}_{\mathbf{p}}^{(1)}(n)$ and our results can thus be related to [5] and [14]. We prove that $\Delta_{12}(n)_{n \geqslant 0}$ and $\mathcal{P}_{\mathbf{y}}^{(1)}(n)_{n \geqslant 0}$ are 2-regular. In Section 7 , we show that the 2-regularity of $\mathcal{P}_{\mathbf{t}}^{(2)}(n)$ follows from the 2-regularity of $\Delta_{12}(n)_{n \geqslant 0}$ and $\mathcal{P}_{\mathbf{y}}^{(1)}(n)_{n \geqslant 0}$.

Finally, in Section 8 we suggest a direction for future work.

\section{Sequences satisfying a reflection symmetry}

The aim of this section is to prove Theorem 4 stated in the introduction. Before proving it in generality, we first examine the sequence satisfying the recurrence for $\ell_{0}=0$ and $c=1$. It will turn out that the general solution can be expressed naturally in terms of this sequence.

Let $A(0)=0$. For each $\ell \geqslant 0$ and $0 \leqslant r \leqslant 2^{\ell}-1$, let

$$
A\left(2^{\ell}+r\right)= \begin{cases}A(r)+1 & \text { if } r \leqslant 2^{\ell-1} \\ A\left(2^{\ell+1}-r\right) & \text { if } r>2^{\ell-1}\end{cases}
$$

The sequence $A(n)_{n \geqslant 0}$ is

$$
0,1,1,2,1,2,2,2,1,2,2,3,2,3,2,2, \ldots
$$

and appears as [16, A007302]. Allouche and Shallit [2, Example 12] identified this sequence as an example of a regular sequence. We include a proof here.

Proposition 6. For all $n \geqslant 0$ we have

$$
\begin{aligned}
A(2 n) & =A(n) \\
A(8 n+1) & =A(4 n+1) \\
A(8 n+3) & =A(2 n+1)+1 \\
A(8 n+5) & =A(2 n+1)+1 \\
A(8 n+7) & =A(4 n+3) .
\end{aligned}
$$

In particular, $A(n)_{n \geqslant 0}$ is 2-regular.

Proof. This proof is typical of many of the proofs throughout the paper. We work by induction on $n$. The case $n=0$ can be checked easily using the first few values of the sequence $A(n)_{n \geqslant 0}$. Therefore, let $n \geqslant 1$ and assume that the recurrence holds for all values less than $n$. Write $n=2^{\ell}+r$ with $\ell \geqslant 0$ and $0 \leqslant r \leqslant 2^{\ell}-1$. 
First let us address the equation $A(2 n)=A(n)$. If $0 \leqslant r \leqslant 2^{\ell-1}$, then

$$
\begin{aligned}
A(2 n) & =A\left(2^{\ell+1}+2 r\right) \\
& =A(2 r)+1 \\
& =A(r)+1 \\
& =A\left(2^{\ell}+r\right) \\
& =A(n) .
\end{aligned}
$$

(by Equation (2))

On the other hand, if $2^{\ell-1}<r<2^{\ell}$, then

$$
\begin{aligned}
A(2 n) & =A\left(2^{\ell+1}+2 r\right) \\
& =A\left(2^{\ell+2}-2 r\right) \\
& =A\left(2^{\ell+1}-r\right) \\
& =A\left(2^{\ell}+r\right) \\
& =A(n) .
\end{aligned}
$$

(by Equation (2))

Next we consider $A(8 n+1)=A(4 n+1)$. If $0 \leqslant r \leqslant 2^{\ell-1}-1$, then

$$
\begin{aligned}
A(8 n+1) & =A\left(2^{\ell+3}+8 r+1\right) \\
& =A(8 r+1)+1 \\
& =A(4 r+1)+1 \\
& =A\left(2^{\ell+2}+4 r+1\right) \\
& =A(4 n+1) .
\end{aligned}
$$

If $2^{\ell-1} \leqslant r<2^{\ell}$, then

$$
\begin{aligned}
A(8 n+1) & =A\left(2^{\ell+3}+8 r+1\right) \\
& =A\left(2^{\ell+4}-8 r-1\right) \\
& =A\left(2^{\ell+4}-8 r-8+7\right) \\
& =A\left(2^{\ell+3}-4 r-4+3\right) \\
& =A\left(2^{\ell+3}-(4 r+1)\right) \\
& =A\left(2^{\ell+2}+4 r+1\right) \\
& =A(4 n+1) .
\end{aligned}
$$

(by induction hypothesis)

(by Equation (2))

The equations for $A(8 n+3), A(8 n+5)$ and $A(8 n+7)$ are handled similarly.

Now we prove Theorem 4. We show that for general $\ell_{0} \geqslant 0$, a sequence $s(n)_{n \geqslant 0}$ satisfying the recurrence can be written in terms of $A(n)_{n \geqslant 0}$.

Proof of Theorem 4. There are $2^{\ell_{0}}$ initial conditions for the recurrence, namely $s(0), \ldots$, $s\left(2^{\ell_{0}}-1\right)$. We claim that most of the $2^{\ell_{0}+2}$ subsequences of the form $s\left(2^{\ell_{0}+2} n+i\right)_{n \geqslant 0}$ 
depend on only one of the initial conditions $s(j)$; each of these subsequences is essentially $A(n)_{n \geqslant 0}, A(4 n+1)_{n \geqslant 0}, A(2 n+1)_{n \geqslant 0}$, or $A(4 n+3)_{n \geqslant 0}$. Furthermore, each of the remaining subsequences is equal to $s\left(2^{\ell_{0}} n+j\right)+c$ for some $j$. More precisely, for $0 \leqslant i \leqslant 2^{\ell_{0}+2}-1$ and $n \geqslant 0$ we have the identity

$$
\begin{aligned}
& s\left(2^{\ell_{0}+2} n+i\right)= \\
& \qquad \begin{cases}c A(n)+s(0) & \text { if } i=0 \\
c A(4 n+1)-c+s(i) & \text { if } 1 \leqslant i \leqslant 2^{\ell_{0}}-1 \\
c A(4 n+1)+s(0) & \text { if } i=2^{\ell_{0}} \\
s\left(2^{\ell_{0}} n+i-2^{\ell_{0}}\right)+c & \text { if } 2^{\ell_{0}}+1 \leqslant i \leqslant 2^{\ell_{0}}+2^{\ell_{0}-1}-1 \\
c A(2 n+1)+s\left(\left|i-2^{\ell_{0}+1}\right|\right) & \text { if } 2^{\ell_{0}}+2^{\ell_{0}-1} \leqslant i \leqslant 2^{\ell_{0}+1}+2^{\ell_{0}-1} \\
s\left(2^{\ell_{0}} n+i-2^{\ell_{0}+1}\right)+c & \text { if } 2^{\ell_{0}+1}+2^{\ell_{0}-1}+1 \leqslant i \leqslant 2^{\ell_{0}+1}+2^{\ell_{0}}-1 \\
c A(4 n+3)+s(0) & \text { if } i=2^{\ell_{0}+1}+2^{\ell_{0}} \\
c A(4 n+3)-c+s\left(2^{\ell_{0}+2}-i\right) & \text { if } 2^{\ell_{0}+1}+2^{\ell_{0}}+1 \leqslant i \leqslant 2^{\ell_{0}+2}-1 .\end{cases}
\end{aligned}
$$

(Note the symmetry among the eight cases, which reflects the symmetry $s\left(2^{\ell}+r\right)=$ $s\left(2^{\ell+1}-r\right)$ of the recurrence for $r>2^{\ell-1}$.) It will follow from this identity that the $\mathbb{Z}$ module generated by the 2-kernel of $s(n)_{n \geqslant 0}$ is generated by the sequences $s\left(2^{\ell} n+j\right)_{n \geqslant 0}$ for $0 \leqslant \ell \leqslant \ell_{0}+1$ and $0 \leqslant j \leqslant 2^{\ell}-1, A(n)_{n \geqslant 0}, A(4 n+1)_{n \geqslant 0}, A(2 n+1)_{n \geqslant 0}, A(4 n+3)_{n \geqslant 0}$, and the constant 1 sequence. In particular, this module is finitely generated.

We prove the identity by induction on $n$. Recall that for all $\ell \geqslant \ell_{0}$ and for all $r$ such that $0 \leqslant r \leqslant 2^{\ell}-1$, we have Equation (1), i.e.,

$$
s\left(2^{\ell}+r\right)= \begin{cases}s(r)+c & \text { if } r \leqslant 2^{\ell-1} \\ s\left(2^{\ell+1}-r\right) & \text { if } r>2^{\ell-1} .\end{cases}
$$

For $n=0$, one uses $A(1)=1$ and $A(3)=2$ to verify that all eight cases of the identity hold. Inductively, let $n \geqslant 1$, and assume the identity is true for all $n^{\prime}<n$. Write $n=2^{\ell}+r$ with $\ell \geqslant 0$ and $0 \leqslant r \leqslant 2^{\ell}-1$.

First we consider the case $0 \leqslant r \leqslant 2^{\ell-1}-1$. For all $i \in\left\{0, \ldots 2^{\ell_{0}+2}-1\right\}$, we have $2^{\ell_{0}+2} r+i \leqslant 2^{\left(\ell_{0}+2+\ell\right)-1}-1$, so

$$
\begin{aligned}
s\left(2^{\ell_{0}+2} n+i\right) & =s\left(2^{\ell_{0}+2+\ell}+\left(2^{\ell_{0}+2} r+i\right)\right) \\
& =s\left(2^{\ell_{0}+2} r+i\right)+c
\end{aligned}
$$

If $1 \leqslant i \leqslant 2^{\ell_{0}}-1$, then the induction hypothesis now gives

$$
\begin{aligned}
s\left(2^{\ell_{0}+2} n+i\right) & =s\left(2^{\ell_{0}+2} r+i\right)+c \\
& =c A(4 r+1)+s(i) \\
& =c\left(A\left(2^{\ell+2}+4 r+1\right)-1\right)+s(i) \\
& =c A(4 n+1)-c+s(i),
\end{aligned}
$$


where we have used $A\left(2^{\ell+2}+4 r+1\right)=A(4 r+1)+1$ from the recurrence for $A(n)$, since $4 r+1 \leqslant 2^{(\ell+2)-1}$. The other seven intervals for $i$ are verified similarly; in each case one applies the induction hypothesis to $s\left(2^{\ell_{0}+2} r+i\right)+c$ and then uses the recurrence for either $A(n)$ or $s(n)$ to raise an argument in $r$ to an argument in $n$.

It remains to consider $2^{\ell-1} \leqslant r \leqslant 2^{\ell}-1$. First we address the case $i=0$. If $r=2^{\ell-1}$ then

$$
\begin{aligned}
s\left(2^{\ell_{0}+2} n+i\right) & =s\left(2^{\ell_{0}+2+\ell}+2^{\ell_{0}+2+\ell-1}\right) \\
& =s\left(2^{\ell_{0}+2+\ell-1}\right)+c \\
& =c A\left(2^{\ell-1}\right)+s(0)+c \\
& =c\left(A\left(2^{\ell}+2^{\ell-1}\right)-1\right)+s(0)+c \\
& =c A(n)+s(0)
\end{aligned}
$$

as desired. Alternatively, if $2^{\ell-1}<r \leqslant 2^{\ell}-1$ then $2^{\ell_{0}+2} r>2^{\left(\ell_{0}+2+\ell\right)-1}$, so

$$
\begin{aligned}
s\left(2^{\ell_{0}+2} n+i\right) & =s\left(2^{\ell_{0}+2+\ell}+2^{\ell_{0}+2} r\right) \\
& =s\left(2^{\ell_{0}+2+\ell+1}-2^{\ell_{0}+2} r\right) \\
& =s\left(2^{\ell_{0}+2}\left(2^{\ell+1}-r\right)+0\right) \\
& =c A\left(2^{\ell+1}-r\right)+s(0) \\
& =c A\left(2^{\ell}+r\right)+s(0) \\
& =c A(n)+s(0) .
\end{aligned}
$$

Therefore it remains to consider $2^{\ell-1} \leqslant r \leqslant 2^{\ell}-1$ for $1 \leqslant i \leqslant 2^{\ell_{0}+2}-1$. In this range we have $2^{\ell_{0}+2} r+i>2^{\left(\ell_{0}+2+\ell\right)-1}$, so

$$
\begin{aligned}
s\left(2^{\ell_{0}+2} n+i\right) & =s\left(2^{\ell_{0}+2+\ell}+\left(2^{\ell_{0}+2} r+i\right)\right) \\
& =s\left(2^{\ell_{0}+2+\ell+1}-2^{\ell_{0}+2} r-i\right) \\
& =s\left(2^{\ell_{0}+2} n^{\prime}+i^{\prime}\right),
\end{aligned}
$$

where $n^{\prime}=2^{\ell+1}-r-1$ and $i^{\prime}=2^{\ell_{0}+2}-i$. We prove the identity for the seven intervals for $i$ using the same steps we have already used several times; we have just applied the recurrence for $s(n)$, so next we use the induction hypothesis, followed by the recurrence for $A(n)$ or $s(n)$, depending on which term appears. For the first interval, if $1 \leqslant i \leqslant 2^{\ell_{0}}-1$, then $2^{\ell_{0}+1}+2^{\ell_{0}}+1 \leqslant i^{\prime} \leqslant 2^{\ell_{0}+2}-1$, so

$$
\begin{array}{rlr}
s\left(2^{\ell_{0}+2} n+i\right) & =s\left(2^{\ell_{0}+2} n^{\prime}+i^{\prime}\right) & \\
& =c A\left(4 n^{\prime}+3\right)-c+s\left(2^{\ell_{0}+2}-i^{\prime}\right) & \text { (by inductive hypothesis) } \\
& =c A\left(2^{\ell+3}-(4 r+1)\right)-c+s(i) & \\
& =c A\left(2^{\ell+2}+4 r+1\right)-c+s(i) & \\
& =c A(4 n+1)-c+s(i) . &
\end{array}
$$

The proofs for the remaining six intervals are routine at this point, so we omit the steps here. 
Example 7. In Section 4, we will use Theorem 4 with $\ell_{0}=2$ to conclude that $\Delta_{0}(n)_{n \geqslant 0}$ and $\mathcal{P}_{\mathbf{x}}^{(1)}(n)_{n \geqslant 0}$ are 2-regular for the period-doubling word. For $\ell_{0}=2$ the value of $s(16 n+i)$ is

$$
s(16 n+i)= \begin{cases}c A(n)+s(0) & \text { if } i=0 \\ c A(4 n+1)-c+s(i) & \text { if } 1 \leqslant i \leqslant 3 \\ c A(4 n+1)+s(0) & \text { if } i=4 \\ s(4 n+1)+c & \text { if } i=5 \\ c A(2 n+1)+s(|i-8|) & \text { if } 6 \leqslant i \leqslant 10 \\ s(4 n+3)+c & \text { if } i=11 \\ c A(4 n+3)+s(0) & \text { if } i=12 \\ c A(4 n+3)-c+s(16-i) & \text { if } 13 \leqslant i \leqslant 15 .\end{cases}
$$

In Section 6, we will use Theorem 4 with $\ell_{0}=1$ to conclude that $\Delta_{12}(n)_{n \geqslant 0}$ is 2-regular for the Thue--Morse word.

\section{About regular sequences and words}

We will often make use of the following composition theorem for a function $F$ defined piecewise on several $k$-automatic sets.

Lemma 8. Let $k \geqslant 2$. Let $P_{1}, \ldots, P_{\ell}: \mathbb{N} \rightarrow\{0,1\}$ be unary predicates that are $k$ automatic. Let $f_{1}, \ldots, f_{\ell}$ be $k$-regular functions. The function $F: \mathbb{N} \rightarrow \mathbb{N}$ defined by

$$
F(n)=\sum_{i=1}^{\ell} f_{i}(n) P_{i}(n)
$$

is k-regular.

Proof. It is a direct consequence of [1, Theorem 2.5]: if $s(n)_{n \geqslant 0}$ and $t(n)_{n \geqslant 0}$ are $k$-regular, then $(s(n)+t(n))_{n \geqslant 0}$ and $(s(n) t(n))_{n \geqslant 0}$ are both $k$-regular sequences. Recall that $k$ automatic sequences are special cases of $k$-regular sequences.

Note that if, for each $n$, there is exactly one $i$ such that $P_{i}(n)=1$, then we can write

$$
F(n)=\left\{\begin{array}{cc}
f_{1}(n) & \text { if } P_{1}(n)=1 \\
f_{2}(n) & \text { if } P_{2}(n)=1 \\
\vdots & \vdots \\
f_{\ell}(n) & \text { if } P_{\ell}(n)=1
\end{array}\right.
$$

This is the setting in which we will apply Lemma 8.

We will also make use of the following classical results. 
Lemma 9. [1, Theorem 2.3] Let $k \geqslant 2$ be an integer. A sequence taking finitely many values is $k$-regular if and only if it is $k$-automatic.

Lemma 10. [1, Corollary 2.4] Let $k, m \geqslant 2$ be integers. If a sequence $s(n)_{n \geqslant 0}$ is $k$-regular, then $(s(n) \bmod m)_{n \geqslant 0}$ is $k$-automatic.

Lemma 11. Let $k \geqslant 2$ be an integer. Let $s(n)_{n \geqslant 0}$ be a sequence. The sequence $s(n)_{n \geqslant 0}$ is $k$-regular if and only if $s(n+1)_{n \geqslant 0}$ is $k$-regular.

Proof. It is a direct consequence of two results stated in [1], namely Theorem 2.6 and its following remark.

Let us now give some definitions about combinatorics on words.

Definition 12. If a word $w$ starts with the letter $a$, then $a^{-1} w$ denotes the word obtained from $w$ by deleting its first letter. Similarly, if a word $w$ ends with the letter $a$, then $w a^{-1}$ denotes the word obtained from $w$ by deleting its last letter. As usual, we let $|w|$ denote the length of the finite word $w$. If $a$ is a letter, we let $|w|_{a}$ denote the number of occurrences of $a$ in $w$. If $w=w_{0} \cdots w_{\ell-1}$, then we let $w^{\mathrm{R}}=w_{\ell-1} \cdots w_{0}$ denote the reversal of $w$. Our convention is that we index letters in an infinite word beginning with 0 .

Since we are interested in $\ell$-abelian complexity, it is natural to consider the following operation that permits us to compare factors of length $\ell$ occurring in an infinite word. Indeed, if two finite words are $\ell$-abelian equivalent, then their $\ell$-block codings are abelian equivalent (but the converse does not hold).

Definition 13. Let $\ell \geqslant 1$. The $\ell$-block coding of the word $\mathbf{w}=w_{0} w_{1} w_{2} \cdots$ over the alphabet $A$ is the word

$$
\operatorname{block}(\mathbf{w}, \ell)=\left(w_{0} \cdots w_{\ell-1}\right)\left(w_{1} \cdots w_{\ell}\right)\left(w_{2} \cdots w_{\ell+1}\right) \cdots\left(w_{j} \cdots w_{j+\ell-1}\right) \cdots
$$

over the alphabet $A^{\ell}$. If $A=\{0, \ldots, r-1\}$, then it is convenient to identify $A^{\ell}$ with the set $\left\{0, \ldots, r^{\ell}-1\right\}$ and each word $w_{0} \cdots w_{\ell-1}$ of length $\ell$ is thus replaced with the integer obtained by reading the word in base $r$, i.e.,

$$
\sum_{i=0}^{\ell-1} w_{i} r^{\ell-1-i}
$$

One can also define accordingly the $\ell$-block coding of a finite word $u$ of length at least $\ell$. The resulting word $\operatorname{block}(u, \ell)$ has length $|u|-\ell+1$.

Example 14. The 2-block codings of 011010011 and 001101101 are respectively 13212013 and 01321321, which are abelian equivalent.

Lemma 15. [13, Lemma 2.3] Let $\ell \geqslant 1$. Two finite words $u$ and $v$ of length at least $\ell-1$ are $\ell$-abelian equivalent if and only if they share the same prefix (resp. suffix) of length $\ell-1$ and the words $\operatorname{block}(u, \ell)$ and $\operatorname{block}(v, \ell)$ are abelian equivalent. 
It is well known that the $\ell$-block coding of a $k$-automatic sequence is again a $k$ automatic sequence [8]. (Note that the operation of $\ell$-block compression that one also encounters in the literature is not the same as the $\ell$-block coding given in Definition 13.)

Example 16. For the period-doubling word p, the 2-block coding is given by

$$
\operatorname{block}(\mathbf{p}, 2)=\phi^{\omega}(1)=12001212120012001200121212001212 \cdots
$$

where $\phi$ is the morphism over $\{0,1,2\}^{*}$ defined by $\phi: 0 \mapsto 12,1 \mapsto 12,2 \mapsto 00$.

Example 17. For the Thue-Morse word $\mathbf{t}$, the 2-block coding is given by

$$
\operatorname{block}(\mathbf{t}, 2)=\nu^{\omega}(1)=132120132012132120121320 \cdots
$$

where $\nu$ is the morphism over $\{0,1,2,3\}^{*}$ defined by $\nu: 0 \mapsto 12,1 \mapsto 13,2 \mapsto 20,3 \mapsto 21$.

\section{Abelian complexity of $\operatorname{block}(\mathrm{p}, 2)$}

We let $\mathbf{x}$ denote block $(\mathbf{p}, 2)=12001212120012001200121212001212 \cdots$, the 2-block coding of $\mathbf{p}$, introduced in Example 16. We consider in this section the abelian complexity of $\mathbf{x}$ and then, in Section 5, we compare $\mathcal{P}_{\mathbf{x}}^{(1)}(n)$ with $\mathcal{P}_{\mathbf{p}}^{(2)}(n)$.

Definition 18. We will make use of functions related to the number of 0's in the factors of $\mathbf{x}$ of a given length. Let $n \in \mathbb{N}$. We let $\max _{0}(n)\left(\operatorname{resp} \min _{0}(n)\right)$ denote the maximum (resp. minimum) number of 0's in a factor of $\mathbf{x}$ of length $n$. Let $\Delta_{0}(n)=\max _{0}(n)-\min _{0}(n)$ be the difference between these two values.

Each of the $\Delta_{0}(n)+1$ integers in the interval $\left[\min _{0}(n), \max _{0}(n)\right]$ is attained as the number of 0 's in some factor of $\mathbf{x}$ of length $n$, since when we slide a window of length $n$ along $\mathbf{x}$ from a factor with $\min _{0}(n)$ zeros to a factor with $\max _{0}(n)$ zeros, the number of 0 's changes by at most 1 per step.

Lemma 19. If $n$ is even, then $\max _{0}(n), \min _{0}(n)$ and $\Delta_{0}(n)$ are even.

Proof. Suppose a factor $w=w_{1} \cdots w_{2 n}$ of $\mathbf{x}$ of even length $2 n$ has an odd number $n_{0}$ of zeros. Since $\phi(0)=\phi(1)=12$ and $\phi(2)=00$, the factor $w$ starts or ends with 0 . Without loss of generality, assume it starts with $w_{1}=0$. Then its last letter must be $w_{2 n}=1$. The words $0 w_{1} \cdots w_{2 n-1}$ and $w_{2} \cdots w_{2 n} 2$ are two factors of length $2 n$ with respectively $n_{0}+1$ and $n_{0}-1$ zeros. Hence, these two factors have even numbers of zeros which are respectively greater than and less than $n_{0}$. The conclusion follows.

We give two related proofs of the 2-regularity of the sequence $\mathcal{P}_{\mathbf{x}}^{(1)}(n)_{n \geqslant 0}$. The first uses the following proposition, which we prove in Section 4.1, together with the fact that $\Delta_{0}(n)_{n \geqslant 0}$ is 2-regular and the two sequences $\left(\Delta_{0}(n) \bmod 2\right)_{n \geqslant 0}$ and $\left(\min _{0}(n) \bmod 2\right)_{n \geqslant 0}$ are 2-automatic (see Section 4.2, Corollary 26). Then the 2-regularity of the sequence $\mathcal{P}_{\mathbf{x}}^{(1)}(n)_{n \geqslant 0}$ will follow from Lemma 8 . 
Proposition 20. For $n \in \mathbb{N}$,

$$
\mathcal{P}_{\mathbf{x}}^{(1)}(n)= \begin{cases}\frac{3}{2} \Delta_{0}(n)+\frac{3}{2} & \text { if } \Delta_{0}(n) \text { is odd } \\ \frac{3}{2} \Delta_{0}(n)+1 & \text { if } \Delta_{0}(n) \text { and } n-\min _{0}(n) \text { are even } \\ \frac{3}{2} \Delta_{0}(n)+2 & \text { if } \Delta_{0}(n) \text { and } n-\min _{0}(n)+1 \text { are even } .\end{cases}
$$

In the second proof, we prove in Section 4.3 the following theorem, which allows us to apply our general result expressed by Theorem 4 .

Theorem 21. Let $\ell \geqslant 2$ and $r$ such that $0 \leqslant r<2^{\ell}-1$. We have

$$
\mathcal{P}_{\mathbf{x}}^{(1)}\left(2^{\ell}+r\right)= \begin{cases}\mathcal{P}_{\mathbf{x}}^{(1)}(r)+3 & \text { if } r \leqslant 2^{\ell-1} \\ \mathcal{P}_{\mathbf{x}}^{(1)}\left(2^{\ell+1}-r\right) & \text { if } r>2^{\ell-1}\end{cases}
$$

In particular, the sequence $\mathcal{P}_{\mathbf{x}}^{(1)}(n)_{n \geqslant 0}$ is 2-regular.

From Theorem 21 we see that $\mathcal{P}_{\mathbf{x}}^{(1)}\left(2^{\ell}\right)=\mathcal{P}_{\mathbf{x}}^{(1)}(0)+3=4$ for all $\ell \geqslant 2$. Additionally, one can check that $\mathcal{P}_{\mathbf{x}}^{(1)}\left(2^{1}\right)=4$.

\subsection{Proof of Proposition 20}

First we mention some properties of factors of the word $\mathbf{x}$.

Lemma 22. The set of factors of $\mathbf{x}$ of length 2 is $\operatorname{Fac}_{\mathbf{x}}(2)=\{00,01,12,20,21\}$.

Proof. It is easy to check that these five words are factors. To prove that they are the only ones, it is enough to check that for any element $u$ in $\{00,01,12,20,21\}$ the three factors of length 2 of $\phi(u)$ are in $\{00,01,12,20,21\}$.

Lemma 23. If $w$ is a factor of $\mathbf{x}$ then $\left.|| w\right|_{1}-|w|_{2} \mid \leqslant 1$. In particular, the letters 1 and 2 alternate in the sequence obtained from $\mathbf{x}$ after erasing the 0 's.

Proof. Let $w$ be a factor of $\mathbf{x}$. There are two cases to consider.

If $w$ can be de-substituted (that is, $w=\phi(v)$ for some $v$ ), then $|w|_{1}=|w|_{2}$ since $|\phi(i)|_{1}=|\phi(i)|_{2}$ for all $i \in\{0,1,2\}$.

If $w$ cannot be de-substituted, then either $w$ has even length and occurs at an odd index in $\mathbf{x}$, or $w$ has odd length. If $w$ has odd length, then deleting either the first or last letter results in a word that can be de-substituted, so $\left.|| w\right|_{1}-|w|_{2} \mid \leqslant 1$. If $w$ has even length and occurs at an odd index, then its first letter is 0 or 2 and its last letter is 0 or 1 ; deleting the first and last letters results in a word that can be de-substituted, so $\left.|| w\right|_{1}-|w|_{2} \mid \leqslant 1$.

Finally, observe that if for all factors of a word $u$, the numbers of two letters $x$ and $y$ differ by at most 1 , then $x$ and $y$ alternate in $u$.

Lemma 24. Let $\tau$ be the morphism defined by $\tau: 0 \mapsto 0,1 \mapsto 2,2 \mapsto 1$. If $w$ is a factor of $\mathbf{x}$, then $\tau(w)^{\mathrm{R}}$ is also a factor of $\mathbf{x}$. 
Proof. We first prove by induction that

$$
\tau(\phi(2 u 1))^{\mathrm{R}}=\phi\left(\tau(12 u)^{\mathrm{R}}\right)
$$

for every factor of the form $2 u 1$ of $\mathbf{x}$.

One checks that this is true for 21 and 2001. If $2 u 1$ is a factor not equal to 21 nor 2001 , then $u$ must contain a 2 and we can write $2 u 1=2 u^{\prime} 12 u^{\prime \prime} 1$ where $2 u^{\prime} 1$ and $2 u^{\prime \prime} 1$ are factors of $\mathbf{x}$. By the induction hypothesis we have

$$
\begin{aligned}
\tau(\phi(2 u 1))^{\mathrm{R}} & =\tau\left(\phi\left(2 u^{\prime} 12 u^{\prime \prime} 1\right)\right)^{\mathrm{R}} \\
& =\tau\left(\phi\left(2 u^{\prime \prime} 1\right)\right)^{\mathrm{R}} \tau\left(\phi\left(2 u^{\prime} 1\right)\right)^{\mathrm{R}} \\
& =\phi\left(\tau\left(12 u^{\prime \prime}\right)^{\mathrm{R}}\right) \phi\left(\tau\left(12 u^{\prime}\right)^{\mathrm{R}}\right) \\
& =\phi\left(\tau\left(12 u^{\prime} 12 u^{\prime \prime}\right)^{\mathrm{R}}\right) \\
& =\phi\left(\tau(12 u)^{\mathrm{R}}\right) .
\end{aligned}
$$

We now prove the lemma by induction on the length of $w$. One can check by hand that the lemma is true for $w$ of length at most 15. Assume the lemma is true for every factor of length at most $n \geqslant 15$, and let $w$ be a factor of length $n+1$. Then $w$ is a factor of $\phi(v)$ for some factor $v$ of $\mathbf{x}$ with $\frac{n+1}{2} \leqslant|v| \leqslant \frac{n+3}{2}$.

Since all factors of length 4 contain a 1 and a 2 , there exists a factor $u$ such that $v$ is a factor of $2 u 1$ and $|2 u 1| \leqslant \frac{n+3}{2}+6$. In particular, $w$ is a factor of $\phi(2 u 1)$ and $\tau(w)^{\mathrm{R}}$ is a factor of $\tau(\phi(2 u 1))^{\mathrm{R}}$. To obtain the conclusion, we just need to show that $\tau(\phi(2 u 1))^{\mathrm{R}}$ is a factor of $\mathbf{x}$.

As by Lemma 22, a 2 is always preceded by a 1 in $\mathbf{x}$, the word $12 u$ is a factor of $\mathbf{x}$ and it has length $|12 u| \leqslant \frac{n+3}{2}+6 \leqslant n$. By induction hypothesis, $\tau(12 u)^{\mathrm{R}}$ is a factor of $\mathbf{x}$. Hence $\phi\left(\tau(12 u)^{\mathrm{R}}\right)$ is also a factor. Finally, using the previous result, $\tau(\phi(2 u 1))^{\mathrm{R}}=\phi\left(\tau(12 u)^{\mathrm{R}}\right)$ is a factor of $\mathbf{x}$.

We can now express $\mathcal{P}_{\mathbf{x}}^{(1)}$ in terms of $\Delta_{0}$.

Proof of Proposition 20. Let $w$ be a factor of $\mathbf{x}$ of length $|w|=n$.

If $|w|-|w|_{0}=|w|_{1}+|w|_{2}$ is even, it follows from Lemma 23 that $|w|_{1}=|w|_{2}$. Therefore every factor of length $n$ containing exactly $|w|_{0}$ zeros is abelian-equivalent to $w$, so the pair $\left(n,|w|_{0}\right)$ determines a unique abelian equivalence class of factors.

If $|w|-|w|_{0}$ is odd, then by Lemma 23 either $|w|_{1}=|w|_{2}+1$ or $|w|_{2}=|w|_{1}+1$. By Lemma 24, there is another factor, $v=\tau(w)^{\mathrm{R}}$, of length $n$, with $|v|_{0}=|w|_{0}$ and $|v|_{1}-|v|_{2}=|w|_{2}-|w|_{1}$. Therefore both possibilities occur, so the number of abelian equivalence classes corresponding to a pair $\left(n,|w|_{0}\right)$ is 2 .

There are $\Delta_{0}(n)+1$ possible values for the number of 0 's in a factor of length $n$. Since 
each value occurs for some factor, we have

$$
\begin{aligned}
\mathcal{P}_{\mathbf{x}}^{(1)}(n) & =\sum_{i=\min _{0}(n)}^{\max _{0}(n)} \begin{cases}1 & \text { if } n-i \text { is even } \\
2 & \text { if } n-i \text { is odd }\end{cases} \\
& =\sum_{j=n-\max _{0}(n)}^{n-\min _{0}(n)} \begin{cases}1 & \text { if } j \text { is even } \\
2 & \text { if } j \text { is odd. }\end{cases}
\end{aligned}
$$

Therefore $\mathcal{P}_{\mathbf{x}}^{(1)}(n)=\frac{3}{2} \Delta_{0}(n)+c(n)$, where $c(n)$ depends only on the parities of $\Delta_{0}(n)$ and $n-\min _{0}(n)$; computing four explicit values allows one to determine the values of $c(n)$ and obtain the equation claimed for $\mathcal{P}_{\mathbf{x}}^{(1)}(n)$.

\section{$4.2 \Delta_{0}(n)_{n \geqslant 0}$ is 2-regular, $\left(\min _{0}(n) \bmod 2\right)_{n \geqslant 0}$ is 2-automatic}

In this section, we prove the following result.

Proposition 25. Let $\ell \geqslant 2$ and $r$ such that $0 \leqslant r<2^{\ell}$. We have

$$
\Delta_{0}\left(2^{\ell}+r\right)= \begin{cases}\Delta_{0}(r)+2 & \text { if } r \leqslant 2^{\ell-1} \\ \Delta_{0}\left(2^{\ell+1}-r\right) & \text { if } r>2^{\ell-1}\end{cases}
$$

Moreover,

$$
\min _{0}\left(2^{\ell}+r\right) \equiv \begin{cases}\min _{0}(r) \quad(\bmod 2) & \text { if } r \leqslant 2^{\ell-1} \\ \min _{0}\left(2^{\ell+1}-r\right)+\Delta_{0}\left(2^{\ell+1}-r\right) \quad(\bmod 2) & \text { if } r>2^{\ell-1}\end{cases}
$$

Before giving the proof, we prove a corollary. The 2-regularity of $\mathcal{P}_{\mathbf{x}}^{(1)}(n)_{n \geqslant 0}$ follows from Proposition 20 and Corollary 26.

Corollary 26. The following statements are true.

- The sequence $\Delta_{0}(n)_{n \geqslant 0}$ is 2-regular.

- The sequence $\left(\Delta_{0}(n) \bmod 2\right)_{n \geqslant 0}$ is 2-automatic.

- The sequence $\left(\min _{0}(n) \bmod 2\right)_{n \geqslant 0}$ is 2-automatic.

Proof. The first assertion is a direct consequence of Proposition 25 and Theorem 4. Note that one can obtain explicit relations satisfied by $\Delta_{0}(n)_{n \geqslant 0}$ from Example 7 . The second assertion follows from Lemma 10.

For the last assertion, for $i \in\{0, \ldots, 31\}$ we prove that, modulo 2 ,

$$
\min _{0}(32 n+i) \equiv \begin{cases}\min _{0}(8 n+1) & \text { if } i \in\{1,5,9,17,25\} \\ \min _{0}(8 n+3) & \text { if } i=11 \\ \min _{0}(8 n+5) & \text { if } i=21 \\ \min _{0}(8 n+7) & \text { if } i \in\{7,15,23,27,31\} \\ 0 & \text { otherwise }\end{cases}
$$


and

$$
\Delta_{0}(32 n+i) \equiv \begin{cases}\Delta_{0}(8 n+1) & \text { if } i \in\{1,5,9,17,25\} \\ \Delta_{0}(8 n+3) & \text { if } i=11 \\ \Delta_{0}(8 n+5) & \text { if } i=21 \\ \Delta_{0}(8 n+7) & \text { if } i \in\{7,15,23,27,31\} \\ 0 & \text { otherwise. }\end{cases}
$$

By Lemma 19, we already know that $\min _{0}(2 n) \equiv \Delta_{0}(2 n) \equiv 0(\bmod 2)$ for any $n \in \mathbb{N}$. Hence the relations above are true for $i$ even. We prove the other relations by induction on $n$. They are true for $n=0$. Let $n>0$ and assume the relations are satisfied for all $n^{\prime}$ such that $0 \leqslant n^{\prime}<n$. We can write $n=2^{\ell}+r$ with $\ell \geqslant 0$ and $0 \leqslant r<2^{\ell}$. Let $i \in\{1, \ldots, 31\}$ be odd.

Assume first that $r<2^{\ell-1}$. We have $32 n+i=2^{\ell+5}+32 r+i$ and $32 r+i<2^{\ell+4}$.

$$
\begin{aligned}
\min _{0}(32 n+i) & \equiv \min _{0}(32 r+i) \\
& \equiv \min _{0}(8 r+j) \\
& \equiv \min _{0}\left(2^{\ell+3}+8 r+j\right) \\
& \equiv \min _{0}(8 n+j) \quad(\bmod 2)
\end{aligned}
$$

for some $j \in\{0, \ldots, 7\}$ according to the relations. A similar reasoning holds for the $\Delta_{0}$ relations.

Assume now that $r \geqslant 2^{\ell-1}$. Since $32 r+i>2^{\ell+4}$, we have

$$
\begin{aligned}
\min _{0}(32 n+i) & \equiv \min _{0}\left(2^{\ell+6}-32 r-i\right)+\Delta_{0}\left(2^{\ell+6}-32 r-i\right) \\
& \equiv \min _{0}\left(32 n^{\prime}+j\right)+\Delta_{0}\left(32 n^{\prime}+j\right) \quad(\bmod 2)
\end{aligned}
$$

with $j=32-i$ and $n^{\prime}=2^{\ell+1}-r-1$. If $i \in\{3,13,19,29\}$, then $j \in\{3,13,19,29\}$. By the induction hypothesis, $\min _{0}\left(32 n^{\prime}+j\right) \equiv \Delta_{0}\left(32 n^{\prime}+j\right) \equiv 0(\bmod 2)$ and we are done.

For the remaining cases, $i, j \notin\{3,13,19,29\}$. As $\min _{0}$ and $\Delta_{0}$ satisfy the same recurrence relations, by the induction hypothesis, there exists $k \in\{1,3,5,7\}$ such that

$$
\begin{aligned}
\min _{0}(32 n+i) & \equiv \min _{0}\left(8 n^{\prime}+k\right)+\Delta_{0}\left(8 n^{\prime}+k\right) \\
& \equiv \min _{0}\left(2^{\ell+4}-(8 r+8-k)\right)+\Delta_{0}\left(2^{\ell+4}-(8 r+8-k)\right) \\
& \equiv \min _{0}\left(2^{\ell+3}+(8 r+8-k)\right) \\
& \equiv \min _{0}(8 n+(8-k)) \quad(\bmod 2) .
\end{aligned}
$$

Observe that the value of $8-k$ is the value given in the relation for $i$. This concludes the proof of the $\min _{0}$ relations. A similar argument works for the $\Delta_{0}$ relations.

We break the proof of Proposition 25 into three parts, covered by Lemmas 27, 29 and 31. We first deal with powers of 2.

Lemma 27. Let $\ell \in \mathbb{N}, \ell \geqslant 1$. We have $\mathcal{P}_{\mathbf{x}}^{(1)}\left(2^{\ell}\right)=4$,

$$
\Delta_{0}\left(2^{\ell}\right)=2, \max _{0}\left(2^{\ell+1}\right)=2^{\ell}-\min _{0}\left(2^{\ell}\right) \text { and } \min _{0}\left(2^{\ell+1}\right)=2^{\ell}-\max _{0}\left(2^{\ell}\right) .
$$


Proof. Recall that $\Psi(w)=\left(|w|_{0},|w|_{1},|w|_{2}\right)$ is the Parikh vector of $w$. We show by induction that

$$
\begin{aligned}
\left\{\Psi(w): w \text { factor of } \mathbf{x} \text { with }|w|=2^{\ell}\right\} \\
=\left\{P_{\ell}+(0,0,0), P_{\ell}+(-2,1,1), P_{\ell}+(-1,1,0), P_{\ell}+(-1,0,1)\right\}
\end{aligned}
$$

and that

$$
\begin{aligned}
& \Psi\left(\phi^{\ell}(0)\right)= \begin{cases}P_{\ell} & \text { if } \ell \text { is even } \\
P_{\ell}+(-2,1,1) & \text { if } \ell \text { is odd }\end{cases} \\
& \Psi\left(\phi^{\ell}(2)\right)= \begin{cases}P_{\ell}+(-2,1,1) & \text { if } \ell \text { is even } \\
P_{\ell} & \text { if } \ell \text { is odd, }\end{cases}
\end{aligned}
$$

where $P_{\ell}=\left(\frac{2^{\ell}+4}{3}, \frac{2^{\ell}-2}{3}, \frac{2^{\ell}-2}{3}\right)$ if $\ell$ is odd and $P_{\ell}=\left(\frac{2^{\ell}+2}{3}, \frac{2^{\ell}-1}{3}, \frac{2^{\ell}-1}{3}\right)$ if $\ell$ is even. Since Parikh vectors of factors of length $2^{\ell}$ can take exactly four values, the conclusion is immediate.

The result is true for $\ell \in\{1,2\}$. Let $\ell>2$ and assume the result holds for $\ell-1$. Let $w$ be a factor of length $2^{\ell}$.

If $w$ can be de-substituted, then $w=\phi(v)$ for some factor $v$ of length $2^{\ell-1}$, and $\Psi(w)=\left(2|v|_{2},|v|_{0}+|v|_{1},|v|_{0}+|v|_{1}\right)$. Using the induction hypothesis, it is easy to check that $\Psi(w)=P_{\ell}$ or $\Psi(w)=P_{\ell}+(-2,1,1)$ and that the equalities for $\Psi\left(\phi^{\ell}(0)\right), \Psi\left(\phi^{\ell}(2)\right)$ are satisfied.

If $w$ cannot be de-substituted, then $w$ occurs at an odd index in $\mathbf{x}$ and $w$ is of the form

$$
0^{-1} \phi(v) 0, \quad 1^{-1} \phi(v) 1, \quad 0^{-1} \phi(v) 1 \quad \text { or } \quad 1^{-1} \phi(v) 0
$$

for some factor $v$ of length $2^{\ell-1}$. If $w$ is of one of the first two forms, then $\Psi(w)=\Psi(\phi(v))$ and $\Psi(w)=P_{\ell}$ or $\Psi(w)=P_{\ell}+(-2,1,1)$ (as in the previous case).

If $w=0^{-1} \phi(v) 1$, then $w$ can also be written as $w=0 \phi(u) 2^{-1}$ for some factor $u$ of length $2^{\ell-1}$. So both Parikh vectors $\Psi(\phi(v))$ and $\Psi(\phi(u))$ belong to $\left\{P_{\ell}, P_{\ell}+(-2,1,1)\right\}$. Since by construction $\phi(v)$ has two more zeros than $\phi(u)$, we obtain $\Psi(\phi(v))=P_{\ell}$ and $\Psi(\phi(u))=P_{\ell}+(-2,1,1)$. Thus $\Psi(w)=\Psi(\phi(v))+(-1,1,0)=P_{\ell}+(-1,1,0)$.

Similarly, if $w=1^{-1} \phi(v) 0$, then $\Psi(w)=P_{\ell}+(-1,0,1)$.

To conclude the proof, we just need to show that these four cases actually occur for all $\ell$. Since $\left\{\Psi\left(\phi^{\ell}(0)\right), \Psi\left(\phi^{\ell}(2)\right)\right\}=\left\{P_{\ell}, P_{\ell}+(-2,1,1)\right\}$, consider all factors of length $2^{\ell}$ occurring between two consecutive occurrences of $\Psi\left(\phi^{\ell}(0)\right)$ and $\Psi\left(\phi^{\ell}(2)\right)$. By continuity ${ }^{2}$, of the number of 0 's, one of these factors must have a Parikh vector equal to $P_{\ell}+(-1,1,0)$ or $P_{\ell}+(-1,0,1)$. Using Lemma 24 , we obtain that $w$ is a factor of length $2^{\ell}$ with $\Psi(w)=$ $P_{\ell}+(-1,1,0)$ if and only if $\tau(w)^{\mathrm{R}}$ is a factor of length $2^{\ell}$ with $\Psi(w)=P_{\ell}+(-1,0,1)$. So all four values actually occur.

To show Lemmas 29 and 31, we first prove the following technical result.

\footnotetext{
${ }^{2}$ We mean by continuity that the number of 0 's is varying by at most 1 between two factors of the same length starting at consecutive indexes.
} 
Lemma 28. Let $u$ be a factor of $\mathbf{x}$ of length $n \geqslant 1$. Let $\max _{2}(n)\left(\right.$ resp. $\left.\min _{2}(n)\right)$ denote the maximum (resp. minimum) of $\left\{|w|_{2}: w\right.$ factor of $\mathbf{x}$ of length $\left.n\right\}$. We have $|u|_{2}=\max _{2}(n)$ if and only if $|\phi(u)|_{0}=\max _{0}(2 n)$, and $|u|_{2}=\min _{2}(n)$ if and only if $|\phi(u)|_{0}=\min _{0}(2 n)$.

Proof. For the first assertion, assume that $|u|_{2}=\max _{2}(n)$ and suppose that $|\phi(u)|_{0}<$ $\max _{0}(2 n)$. Note that $|\phi(u)|_{0}=2|u|_{2}$ by definition of $\phi$. Let $v$ be a factor of length $2 n$ such that $|v|_{0}=\max _{0}(2 n)$, which is even by Lemma 19. In addition, we can assume that $v$ starts with 00. Indeed, if it is not the case, then either $v$ starts with 01 and ends with 0 , or $v$ is of the form $t 00 s$ where $t$ does not contain any zero. In the first case, we can consider the word $0 v 0^{-1}$ that starts with 00 and has $\max _{0}(2 n)$ zeros. In the second case, we can consider the word $00 \mathrm{~s} w$ for some $w$ with $|w|=|t|$. This factor has also $\max _{0}(2 n)$ zeros. Therefore $v$ can be de-substituted. So $v=\phi(z)$ and $|z|_{2}=\frac{1}{2}|v|_{0}>|u|_{2}$, which is a contradiction.

For the other direction, assume $|\phi(u)|_{0}=\max _{0}(2 n)$ and suppose $|u|_{2}$ does not maximize the number of 2's. Then there exists a factor $v$ of length $n$ such that $|v|_{2}=\max _{2}(n)$. Hence,

$$
|\phi(v)|_{0}=2|v|_{2}>2|u|_{2}=|\phi(u)|_{0}=\max _{0}(2 n),
$$

which is a contradiction. Similar arguments hold for the second assertion.

Lemma 29. If $\ell \geqslant 2$ and $0 \leqslant r \leqslant 2^{\ell-1}$, then

$$
\begin{aligned}
\max _{0}\left(2^{\ell}+r\right) & =\max _{0}\left(2^{\ell}\right)+\max _{0}(r), \\
\min _{0}\left(2^{\ell}+r\right) & =\min _{0}\left(2^{\ell}\right)+\min _{0}(r) .
\end{aligned}
$$

Proof. We work by induction on $\ell$. One checks the case $\ell=2$. Let $\ell>2$ and assume the statements are true for $\ell-1$. Let $r$ such that $0 \leqslant r \leqslant 2^{\ell-1}$.

Assume first that $r$ is even. We shall exhibit a factor of length $2^{\ell}+r$ that has $\max _{0}\left(2^{\ell}\right)+\max _{0}(r)$ zeros and maximizes the number of 0's. By the induction hypothesis, the result is true for $2^{\ell-1}+r / 2$. So there exists a factor $u$ of length $2^{\ell-1}+r / 2$ with $\min _{0}\left(2^{\ell-1}+r / 2\right)=\min _{0}\left(2^{\ell-1}\right)+\min _{0}(r / 2)$ zeros. In addition, we can assume that $u$ maximizes the number of 2 's. Indeed, since $|u|_{0}=\min _{0}\left(2^{\ell-1}+r / 2\right),|u|_{1}+|u|_{2}$ is maximal among all factors of length $2^{\ell-1}+r / 2$. If the number of 1 and 2 in $u$ is even, then $|u|_{2}=|u|_{1}$ is maximal. Otherwise, either $|u|_{2}=|u|_{1}+1$ and $|u|_{2}$ is maximal, or $|u|_{2}=|u|_{1}-1$ and $u$ does not maximize the number of 2's. In the last case, by Lemma 24, we can consider the factor $\tau(u)^{\mathrm{R}}$ which satisfies $\left|\tau(u)^{\mathrm{R}}\right|_{0}=|u|_{0}$ and $\left|\tau(u)^{\mathrm{R}}\right|_{2}=|u|_{1}$. Hence, $\tau(u)^{\mathrm{R}}$ minimizes the number of 0's and maximizes the number of 2's.

Let us write $u=v w$ with $|v|=2^{\ell-1}$ and $|w|=r / 2$. Then, as $|v|_{0}+|w|_{0}=|u|_{0}=$ $\min _{0}\left(2^{\ell-1}\right)+\min _{0}(r / 2)$, the words $v$ and $w$ minimize the number of 0 's for words of their respective lengths. The word $v$ maximizes also the number of 2's for factors of length $2^{\ell-1}$ because $|v|$ and $|v|_{0}=\min _{0}\left(2^{\ell-1}\right)$ are even by Lemma 27 and so is $|v|_{1}+|v|_{2}$. Since $u$ maximizes the number of 2's and $|v|_{2}=|v|_{1}$, the word $w$ also maximizes the number of 2 's. Hence, by Lemma 28, $\phi(u), \phi(v)$ and $\phi(w)$ maximize the number of 0's for words of their respective lengths. Thus,

$$
\max _{0}\left(2^{\ell}+r\right)=|\phi(u)|_{0}=|\phi(v)|_{0}+|\phi(w)|_{0}=\max _{0}\left(2^{\ell}\right)+\max _{0}(r) .
$$


If $r$ is odd, we still have $0 \leqslant r-1 \leqslant r+1 \leqslant 2^{\ell-1}$ and we can use the previous results:

$$
\begin{aligned}
& \max _{0}\left(2^{\ell}+r-1\right)=\max _{0}\left(2^{\ell}\right)+\max _{0}(r-1), \\
& \max _{0}\left(2^{\ell}+r+1\right)=\max _{0}\left(2^{\ell}\right)+\max _{0}(r+1) .
\end{aligned}
$$

Note that $\max _{0}$ is even for even values and can only grow by 0 or 1 . So there are two cases to consider: either $\max _{0}\left(2^{\ell}+r+1\right)=\max _{0}\left(2^{\ell}+r-1\right)$ or $\max _{0}\left(2^{\ell}+r+1\right)=$ $\max _{0}\left(2^{\ell}+r-1\right)+2$.

If the two maxima are equal, then $\max _{0}(r+1)=\max _{0}(r-1), \max _{0}\left(2^{\ell}+r\right)=$ $\max _{0}\left(2^{\ell}+r-1\right)$ and $\max _{0}(r)=\max _{0}(r-1)$, and we are done. Otherwise, the two maxima differ by 2 , and then $\max _{0}(r+1)=\max _{0}(r-1)+2, \max _{0}\left(2^{\ell}+r\right)=\max _{0}\left(2^{\ell}+r-1\right)+1$ and $\max _{0}(r)=\max _{0}(r-1)+1$, and we are done.

A similar proof shows that $\min _{0}\left(2^{\ell}+r\right)=\min _{0}\left(2^{\ell}\right)+\min _{0}(r)$.

Lemma 31 will follow directly from the following lemma.

Lemma 30. If $\ell \geqslant 2$ and $2^{\ell-1} \leqslant r \leqslant 2^{\ell}$, then

$$
\begin{aligned}
\max _{0}\left(2^{\ell+1}\right) & =\max _{0}\left(2^{\ell}+r\right)+\min _{0}\left(2^{\ell}-r\right), \\
\min _{0}\left(2^{\ell+1}\right) & =\min _{0}\left(2^{\ell}+r\right)+\max _{0}\left(2^{\ell}-r\right) .
\end{aligned}
$$

Moreover, there is a factor of length $2^{\ell+1}$ maximizing (resp. minimizing) the number of 0 's such that the prefix of length $2^{\ell}+r$ also maximizes (resp. minimizes) the number of 0 's. In addition, the first equality $\max _{0}\left(2^{\ell+1}\right)=\max _{0}\left(2^{\ell}+r\right)+\min _{0}\left(2^{\ell}-r\right)$ holds even if $\ell=1$.

Proof. We proceed by induction on $\ell$. One checks that the results are true for $\ell=2$ and, for the first equality, for $\ell=1$. Let $\ell>2$ and assume both equalities hold for $\ell-1$. Let $r$ such that $2^{\ell-1} \leqslant r \leqslant 2^{\ell}$.

Assume first that $r$ is even. By the induction hypothesis, there exists a factor $u=v w$ of length $2^{\ell}$ such that

$$
|u|_{0}=\min _{0}\left(2^{\ell}\right)=\min _{0}\left(2^{\ell-1}+r / 2\right)+\max _{0}\left(2^{\ell-1}-r / 2\right),
$$

$|v|=2^{\ell-1}+r / 2$ and $v$ minimizes the number of 0 's. Hence, $|v|_{0}=\min _{0}\left(2^{\ell-1}+r / 2\right)$ and $|w|_{0}=\max _{0}\left(2^{\ell-1}-r / 2\right)$.

Observe that $u$ maximizes the number of 2's as $|u|$ and $|u|_{0}=\min _{0}\left(2^{\ell}\right)$ are even. In addition, we can assume that $v$ also maximizes the number of 2's. Indeed, if $v$ is of even length, $|v|_{0}=\min _{0}\left(2^{\ell-1}+r / 2\right)$ implies $|v|_{2}$ is maximal. If $v$ is of odd length and $v$ does not maximize the number of 2's, then it ends with 1 . Thus, $v$ is followed by a 2 . In particular, $v$ occurs at an even index in $\mathbf{x}$. So is $u$ and $u 12$ or $u 00$ is a factor of $\mathbf{x}$. If $u 12$ is a factor, then consider, instead of $u, u^{\prime}=z^{-1} u 1$ where $z$ denotes the first letter of $u$. In that case, the prefix of length $2^{\ell-1}+r / 2$ of $u^{\prime}$ is $z^{-1} v 2$. It still minimizes the number of 0 's and now maximizes the number of 2 's. Assume now that $u 00$ is a factor. Observe that $\mathbf{x}$ is the fixed point of $\phi$. So it is also the fixed point of $\phi^{2}$. Therefore, $\mathbf{x}$ is a concatenation 
of blocks of length 4 of the form $\phi^{2}(0)=\phi^{2}(1)=1200$ and $\phi^{2}(2)=1212$. Since $u 00$ is a factor of $\mathbf{x}$, the only extension of this factor is $12 u 00$ as $|u|=2^{\ell} \equiv 0(\bmod 4)$. Consider then $u^{\prime}=2 u 2^{-1}$.

Since $|u|_{1}=|u|_{2}$ and $|v|_{2} \geqslant|v|_{1},|w|_{1} \geqslant|w|_{2}$. Thus, as $|w|_{0}=\max _{0}\left(2^{\ell-1}-r / 2\right), w$ minimizes the number of 2's. By Lemma 28, we obtain $|\phi(u)|_{0}=\max _{0}\left(2^{\ell+1}\right),|\phi(v)|_{0}=$ $\max _{0}\left(2^{\ell}+r\right),|\phi(w)|_{0}=\min _{0}\left(2^{\ell}-r\right)$. So

$$
\begin{aligned}
\max _{0}\left(2^{\ell+1}\right) & =|\phi(u)|_{0}=|\phi(v)|_{0}+|\phi(w)|_{0} \\
& =\max _{0}\left(2^{\ell}+r\right)+\min _{0}\left(2^{\ell}-r\right) .
\end{aligned}
$$

We can show similarly that $\min _{0}\left(2^{\ell+1}\right)=\min _{0}\left(2^{\ell}+r\right)+\max _{0}\left(2^{\ell}-r\right)$. Note that in this case, we can assume that the factor $u$ with $|u|_{0}=\max _{0}\left(2^{\ell}\right)$, given by the induction hypothesis, starts with 00 as in the proof of Lemma 28.

Assume now that $r$ is odd. Then $2^{\ell-1} \leqslant r-1<r+1 \leqslant 2^{\ell}$ and we can apply the previous result:

$$
\begin{aligned}
\max _{0}\left(2^{\ell+1}\right) & =\max _{0}\left(2^{\ell}+r-1\right)+\min _{0}\left(2^{\ell}-r+1\right) \\
& =\max _{0}\left(2^{\ell}+r+1\right)+\min _{0}\left(2^{\ell}-r-1\right) .
\end{aligned}
$$

Since $\max _{0}$ is even for even values and can only grow by 0 or 1 , there are two cases to consider: either $\max _{0}\left(2^{\ell}+r-1\right)=\max _{0}\left(2^{\ell}+r+1\right)$ or $\max _{0}\left(2^{\ell}+r-1\right)+2=\max _{0}\left(2^{\ell}+r+1\right)$.

If the two maxima are equal, then $\min _{0}\left(2^{\ell}-r+1\right)=\min _{0}\left(2^{\ell}-r-1\right)=\min _{0}\left(2^{\ell}-r\right)$ and $\max _{0}\left(2^{\ell}+r\right)=\max _{0}\left(2^{\ell}+r-1\right)$, and we are done. Otherwise, the two maxima differ by 2 , and then $\min _{0}\left(2^{\ell}-r+1\right)-2=\min _{0}\left(2^{\ell}-r-1\right)$. So $\max _{0}\left(2^{\ell}+r\right)=\max _{0}\left(2^{\ell}+r-1\right)+1$ and $\min _{0}\left(2^{\ell}-r\right)=\min _{0}\left(2^{\ell}-r+1\right)-1$, and we are done. Using similar argument, we can conclude that $\min _{0}\left(2^{\ell+1}\right)=\min _{0}\left(2^{\ell}+r\right)+\max _{0}\left(2^{\ell}-r\right)$.

For the construction of the factors, one can construct them using the factors $\phi(u)$ and $\phi\left(u^{\prime}\right)$ given for $r-1$ and $r+1$ in the previous construction. We consider the same two cases as before.

If the maxima are equal, then $\max _{0}\left(2^{\ell}+r\right)=\max _{0}\left(2^{\ell}+r-1\right)$. By construction, $\phi(u)$ has a prefix $\phi(v)$ of length $2^{\ell}+r-1$, maximizing the number of 0 's. The letter $z$ following the prefix $\phi(v)$ in $\phi(u)$ is not a 0 . Otherwise, $\phi(v) 0$ would be a factor of length $2^{\ell}+r$ with $\max _{0}\left(2^{\ell}+r\right)+1$ zeros, which is a contradiction. Hence, $\phi(v) z$ is a prefix of length $2^{\ell}+r$ of $\phi(u)$ that maximizes the number of 0 's.

If $\max _{0}\left(2^{\ell}+r-1\right)+2=\max _{0}\left(2^{\ell}+r+1\right)$, then $\max _{0}\left(2^{\ell}+r\right)=\max _{0}\left(2^{\ell}+r+1\right)-1$. By construction, $\phi\left(u^{\prime}\right)$ has a prefix $\phi\left(v^{\prime}\right)$ of length $2^{\ell}+r+1$, maximizing the number of 0 's. This prefix must end with 0. Otherwise, deleting the last letter of $\phi\left(v^{\prime}\right)$ would give a factor of length $2^{\ell}+r$ with $\max _{0}\left(2^{\ell}+r+1\right)=\max _{0}\left(2^{\ell}+r\right)+1$ zeros, which is a contradiction. Hence, $\phi\left(v^{\prime}\right) 0^{-1}$ is a prefix of length $2^{\ell}+r$ of $\phi\left(u^{\prime}\right)$ that maximizes the number of 0's.

A similar construction yields a factor of length $2^{\ell+1}$ minimizing the number of 0 's such that the prefix of length $2^{\ell}+r$ also minimizes the number of 0 's.

The previous lemma permits us to reformulate some relations between the two sequences $\max _{0}(n)_{n \geqslant 0}$ and $\min _{0}(n)_{n \geqslant 0}$. 
Lemma 31. If $\ell \geqslant 2$ and $2^{\ell-1} \leqslant r \leqslant 2^{\ell}$, then

$$
\begin{aligned}
\max _{0}\left(2^{\ell}+r\right) & =2^{\ell}-\min _{0}\left(2^{\ell+1}-r\right), \\
\min _{0}\left(2^{\ell}+r\right) & =2^{\ell}-\max _{0}\left(2^{\ell+1}-r\right) .
\end{aligned}
$$

The first equality holds even if $\ell=1$.

Proof. One can check the first equality for $\ell=1$. Let $\ell \geqslant 2$ and $r$ such that $2^{\ell-1} \leqslant r \leqslant 2^{\ell}$. From the previous lemma, we have

$$
\max _{0}\left(2^{\ell}+r\right)=\max _{0}\left(2^{\ell+1}\right)-\min _{0}\left(2^{\ell}-r\right) .
$$

Note that, by Lemma 27 , we have $\max _{0}\left(2^{\ell+1}\right)=2^{\ell}-\min _{0}\left(2^{\ell}\right)$. Moreover, by Lemma 29, since $0 \leqslant 2^{\ell}-r \leqslant 2^{\ell}$, we get

$$
\min _{0}\left(2^{\ell}\right)+\min _{0}\left(2^{\ell}-r\right)=\min _{0}\left(2^{\ell}+2^{\ell}-r\right) .
$$

Since similar relations hold when exchanging $\min _{0}$ and $\max _{0}$, the conclusion follows.

The proof of Proposition 25 about the reflection relation satisfied by $\Delta_{0}(n)$ and the recurrence relation of $\min _{0}(n)$ is now immediate.

Proof of Proposition 25. Let $\ell \geqslant 2$. For $r$ such that $0 \leqslant r \leqslant 2^{\ell-1}$, subtracting the two relations provided by Lemma 29 gives $\Delta_{0}\left(2^{\ell}+r\right)=\Delta_{0}\left(2^{\ell}\right)+\Delta_{0}(r)$ and we can conclude using the first relation given in Lemma 27, $\Delta_{0}\left(2^{\ell}\right)=2$. Furthermore, $\min _{0}\left(2^{\ell}+r\right) \equiv$ $\min _{0}\left(2^{\ell}\right)+\min _{0}(r)(\bmod 2)$ by Lemma 29 . The expression for $\min _{0}\left(2^{\ell}+r\right)$ follows since $\min _{0}\left(2^{\ell}\right) \equiv 0(\bmod 2)$ by Lemma 27 .

For $2^{\ell-1}<r<2^{\ell}$, subtracting the two relations provided by Lemma 31 permits us to conclude the proof of the expression claimed for $\Delta_{0}\left(2^{\ell}+r\right)$. Moreover, using Lemma 31, we get

$$
\begin{aligned}
\min _{0}\left(2^{\ell}+r\right) & \equiv \max _{0}\left(2^{\ell+1}-r\right) \quad(\bmod 2) \\
& \equiv \min _{0}\left(2^{\ell+1}-r\right)+\Delta_{0}\left(2^{\ell+1}-r\right) \quad(\bmod 2) . \square
\end{aligned}
$$

\subsection{Another proof of the 2-regularity of $\mathcal{P}_{\mathrm{x}}^{(1)}(n)_{n \geqslant 0}$}

In this section we prove the 2-regularity of the abelian complexity $\mathcal{P}_{\mathbf{x}}^{(1)}(n)_{n \geqslant 0}$ in a second way, by proving Theorem 21. The proof makes use of Propositions 20 and 25.

Proof of Theorem 21. If $2^{\ell-1} \leqslant r \leqslant 2^{\ell}$, since all the conditions in Proposition 20 are equivalent whether considering $2^{\ell}+r$ or $2^{\ell+1}-r$, we have

$$
\mathcal{P}_{\mathbf{x}}^{(1)}\left(2^{\ell}+r\right)=\mathcal{P}_{\mathbf{x}}^{(1)}\left(2^{\ell+1}-r\right) .
$$

Assume now that $0 \leqslant r \leqslant 2^{\ell-1}$. If $\Delta_{0}\left(2^{\ell}+r\right)$ is odd, $\Delta_{0}(r)$ is also odd by Proposition 25 . By Proposition 20, we have $\mathcal{P}_{\mathbf{x}}^{(1)}\left(2^{\ell}+r\right)=\frac{3}{2}\left(\Delta_{0}\left(2^{\ell}+r\right)+1\right)$ and $\mathcal{P}_{\mathbf{x}}^{(1)}(r)=\frac{3}{2}\left(\Delta_{0}(r)+1\right)$. By 
Proposition 25, we have $\Delta_{0}\left(2^{\ell}+r\right)=\Delta_{0}(r)+2$. Putting these three equalities together, we get $\mathcal{P}_{\mathbf{x}}^{(1)}\left(2^{\ell}+r\right)=\mathcal{P}_{\mathbf{x}}^{(1)}(r)+3$.

The other cases can be done similarly. If $\Delta_{0}\left(2^{\ell}+r\right)$ and $2^{\ell}+r-\min _{0}\left(2^{\ell}+r\right)$ are even, then $\Delta_{0}(r)$ and $r-\min _{0}(r)$ are even and

$$
\begin{aligned}
\mathcal{P}_{\mathbf{x}}^{(1)}\left(2^{\ell}+r\right) & =\frac{3}{2} \Delta_{0}\left(2^{\ell}+r\right)+1 & & \text { (by Proposition 20) } \\
& =\frac{3}{2}\left(\Delta_{0}(r)+2\right)+1 & & \text { (by Proposition 25) } \\
& =\mathcal{P}_{\mathbf{x}}^{(1)}(r)+3 & & \text { (by Proposition 20). }
\end{aligned}
$$

If $\Delta_{0}\left(2^{\ell}+r\right)$ is even and $2^{\ell}+r-\min _{0}\left(2^{\ell}+r\right)$ is odd, then $\Delta_{0}(r)$ is even and $r-\min _{0}(r)$ is odd. Then

$$
\begin{aligned}
\mathcal{P}_{\mathbf{x}}^{(1)}\left(2^{\ell}+r\right) & =\frac{3}{2} \Delta_{0}\left(2^{\ell}+r\right)+2 & & \text { (by Proposition 20) } \\
& =\frac{3}{2}\left(\Delta_{0}(r)+2\right)+2 & & (\text { by Proposition 25) } \\
& =\mathcal{P}_{\mathbf{x}}^{(1)}(r)+3 & & (\text { by Proposition 20). }
\end{aligned}
$$

One can prove the following result in a manner similar to the proof of Theorem 4 . There may be simpler recurrences, but these relations exhibit the same symmetry as in Theorem 4.

Theorem 32. The abelian complexity sequence $\mathcal{P}_{\mathbf{x}}^{(1)}(n)_{n \geqslant 0}$ of the 2-block coding of the period-doubling word satisfies the following relations.

$$
\begin{aligned}
\mathcal{P}_{\mathbf{x}}^{(1)}(8 n) & =\mathcal{P}_{\mathbf{x}}^{(1)}(2 n) \\
4 \mathcal{P}_{\mathbf{x}}^{(1)}(8 n+1) & =-2 \mathcal{P}_{\mathbf{x}}^{(1)}(2 n+1)+7 \mathcal{P}_{\mathbf{x}}^{(1)}(4 n+1)-2 \mathcal{P}_{\mathbf{x}}^{(1)}(4 n+2)+\mathcal{P}_{\mathbf{x}}^{(1)}(4 n+3) \\
4 \mathcal{P}_{\mathbf{x}}^{(1)}(8 n+2) & =-6 \mathcal{P}_{\mathbf{x}}^{(1)}(2 n+1)+9 \mathcal{P}_{\mathbf{x}}^{(1)}(4 n+1)-2 \mathcal{P}_{\mathbf{x}}^{(1)}(4 n+2)+3 \mathcal{P}_{\mathbf{x}}^{(1)}(4 n+3) \\
4 \mathcal{P}_{\mathbf{x}}^{(1)}(8 n+3) & =-6 \mathcal{P}_{\mathbf{x}}^{(1)}(2 n+1)+5 \mathcal{P}_{\mathbf{x}}^{(1)}(4 n+1)+2 \mathcal{P}_{\mathbf{x}}^{(1)}(4 n+2)+3 \mathcal{P}_{\mathbf{x}}^{(1)}(4 n+3) \\
\mathcal{P}_{\mathbf{x}}^{(1)}(8 n+4) & =\mathcal{P}_{\mathbf{x}}^{(1)}(4 n+2) \\
4 \mathcal{P}_{\mathbf{x}}^{(1)}(8 n+5) & =-6 \mathcal{P}_{\mathbf{x}}^{(1)}(2 n+1)+3 \mathcal{P}_{\mathbf{x}}^{(1)}(4 n+1)+2 \mathcal{P}_{\mathbf{x}}^{(1)}(4 n+2)+5 \mathcal{P}_{\mathbf{x}}^{(1)}(4 n+3) \\
4 \mathcal{P}_{\mathbf{x}}^{(1)}(8 n+6) & =-6 \mathcal{P}_{\mathbf{x}}^{(1)}(2 n+1)+3 \mathcal{P}_{\mathbf{x}}^{(1)}(4 n+1)-2 \mathcal{P}_{\mathbf{x}}^{(1)}(4 n+2)+9 \mathcal{P}_{\mathbf{x}}^{(1)}(4 n+3) \\
4 \mathcal{P}_{\mathbf{x}}^{(1)}(8 n+7) & =-2 \mathcal{P}_{\mathbf{x}}^{(1)}(2 n+1)+\mathcal{P}_{\mathbf{x}}^{(1)}(4 n+1)-2 \mathcal{P}_{\mathbf{x}}^{(1)}(4 n+2)+7 \mathcal{P}_{\mathbf{x}}^{(1)}(4 n+3)
\end{aligned}
$$

\section{$5 \quad 2$-abelian complexity of the period-doubling word}

To prove the 2-regularity of $\mathcal{P}_{\mathbf{p}}^{(2)}(n)_{n \geqslant 0}$, the aim of this section is to express the 2-abelian complexity $\mathcal{P}_{\mathbf{p}}^{(2)}$ in terms of the 1-abelian complexity $\mathcal{P}_{\mathbf{x}}^{(1)}$ and the following additional 2-regular functions.

Definition 33. We define the max-jump function $\mathrm{MJ}_{0}: \mathbb{N} \rightarrow\{0,1\}$ by $\mathrm{MJ}_{0}(0)=0$ and, for $n \geqslant 1$,

$$
\operatorname{MJ}_{0}(n)= \begin{cases}1 & \text { if } \max _{0}(n)>\max _{0}(n-1) \\ 0 & \text { otherwise }\end{cases}
$$


i.e., $\operatorname{MJ}_{0}(n)=1$ when the function $\max _{0}$ increases. Similarly, let $\mathrm{mj}_{0}: \mathbb{N} \rightarrow\{0,1\}$ be the min-jump function defined by

$$
\operatorname{mj}_{0}(n)= \begin{cases}1 & \text { if } \min _{0}(n+1)>\min _{0}(n) \\ 0 & \text { otherwise }\end{cases}
$$

Since $\max _{0}(n)$ and $\min _{0}(n)$ are non-decreasing, we can write

$$
\begin{aligned}
\operatorname{MJ}_{0}(n+1) & =\max _{0}(n+1)-\max _{0}(n) \\
\operatorname{mj}_{0}(n) & =\min _{0}(n+1)-\min _{0}(n)
\end{aligned}
$$

The relationship between these sequences and $\mathcal{P}_{\mathbf{p}}^{(2)}$ and $\mathcal{P}_{\mathbf{x}}^{(1)}$ is stated in the following result.

Proposition 34. Let $n \geqslant 1$ be an integer. Then

$$
\mathcal{P}_{\mathbf{p}}^{(2)}(n+1)-\mathcal{P}_{\mathbf{x}}^{(1)}(n)= \begin{cases}0 & \text { if } n \text { is odd } \\ \frac{\Delta_{0}(n)}{2}+1-\mathrm{MJ}_{0}(n)-\mathrm{mj}_{0}(n) & \text { if } n \text { is even } .\end{cases}
$$

We require several preliminary results.

Proposition 35. Let $u$ and $v$ be factors of $\mathbf{p}$ of length $n$. Let $u^{\prime}$ and $v^{\prime}$ be the 2-block codings of $u$ and $v$. The factors $u$ and $v$ are 2-abelian equivalent if and only if $u^{\prime}$ and $v^{\prime}$ are abelian equivalent and either $u^{\prime}$ and $v^{\prime}$ both start with 2 or none of them start with 2 .

Proof. By Lemma 15, $u$ and $v$ are 2-abelian equivalent if and only if they start with the same letter and have the same number of factors 00,01 and 10 . The number of 00 (respectively 01 and 10) in $u$ is exactly the number of 0 (resp. 1 and 2) in $u^{\prime}$. Moreover, $u$ starts with 0 (resp. by 1 ) if and only if $u^{\prime}$ starts with 0 or 1 (resp. by 2). Therefore, $u$ and $v$ are 2-abelian equivalent if and only if $u^{\prime}$ and $v^{\prime}$ are abelian equivalent and both start with 2 or none of them start with 2.

To compute $\mathcal{P}_{\mathbf{p}}^{(2)}$, we will use the abelian complexity of $\mathbf{x}=\operatorname{block}(\mathbf{p}, 2), \mathcal{P}_{\mathbf{x}}^{(1)}$, and study when an abelian equivalence class of $\mathbf{x}$ splits into two 2-abelian equivalence classes of $\mathbf{p}$, or in other words, study when two abelian equivalent factors of $\mathbf{x}$ can start, respectively, with 2 and with 0 or 1 . If the class does not split, we say that it leads to only one class.

Lemma 36. Let $\mathcal{X}$ be an abelian equivalence class of factors of length $n$ of $\mathbf{x}$. If the number of 1 's in an element of $\mathcal{X}$ differs from the number of 2 's, then $\mathcal{X}$ leads to only one 2-abelian equivalence class of $\mathbf{p}$.

Proof. It is enough to prove that if an element of $\mathcal{X}$ starts with 2, all the other elements of $\mathcal{X}$ start with 2. If $u$ starts with 2, then all the elements of $\mathcal{X}$ have more 2's than 1's. But any factor with more 2's than 1's starts with a 2 .

Corollary 37. If $n$ is odd, $\mathcal{P}_{\mathbf{p}}^{(2)}(n+1)=\mathcal{P}_{\mathbf{x}}^{(1)}(n)$. 
Proof. Let $\mathcal{X}$ be an abelian equivalence class of factors of odd length $n$. If no element of $\mathcal{X}$ starts with a $2, \mathcal{X}$ leads to only one 2 -abelian equivalence class of factors of $\mathbf{p}$. So assume that there is a factor $u$ in $\mathcal{X}$ starting with 2. Since $n$ is odd, we can write $u=2 \phi\left(u^{\prime}\right)$. Then the number of 0 's in $u$ is even and there is a different number of 2's than 1's. By Lemma $36, \mathcal{X}$ again leads to a unique 2 -abelian equivalence class of $\mathbf{p}$.

Corollary 38. Let $\mathcal{X}$ be an abelian equivalence class of factors of $\mathbf{x}$ of even length $n$ with an odd number of zeros. Then $\mathcal{X}$ leads to only one 2-abelian equivalence class of $\mathbf{p}$.

Proof. Factors in $\mathcal{X}$ have an odd number of 1's and 2's counted together, so the number of 1 's and the number of 2's are different and we can apply Lemma 36.

Thus, an abelian equivalence class $\mathcal{X}$ of factors of length $n$ of $\mathbf{x}$ can possibly lead to two 2-abelian equivalence classes of factors of length $n+1$ of $\mathbf{p}$ only if $n$ is even and if there are an even number of zeros in $\mathcal{X}$. In most cases $\mathcal{X}$ will indeed lead to two different equivalence classes. The exceptions are identified by the following lemma.

Lemma 39. Let $n$ be a positive even integer and $n_{0}$ such that $\min _{0}(n) \leqslant n_{0} \leqslant \max _{0}(n)$. Let $\mathcal{X}$ be an abelian equivalence class of factors of $\mathbf{x}$ of length $n$ with exactly $n_{0}$ zeros.

- We have $n_{0}=\max _{0}(n)$ and $\mathrm{MJ}_{0}(n)=1$ if and only if every factor $u$ in $\mathcal{X}$ can be written as $u=00 u^{\prime} 00$.

- We have $n_{0}=\min _{0}(n)$ and $\operatorname{mj}_{0}(n)=1$ if and only if every factor $u$ in $\mathcal{X}$ is preceded and followed only by 00 .

Proof. We start by proving the first part of the lemma. Assume that all the elements of $\mathcal{X}$ have the form $00 u^{\prime} 00$. In particular, $n_{0}$ is even. If $n_{0} \neq \max _{0}(n)$, it means that there is a factor $v$ of length $n$ with $n_{0}+1$ zeros. Indeed, sliding a window of length $n$ from a word of $\mathcal{X}$ to a factor with $\max _{0}(n)$ zeros gives factors with all possibilities between $n_{0}$ and $\max _{0}(n)$ for the number of zeros. Since $|v|_{0}$ is odd and $n$ is even, we must have $v=0 \phi\left(v^{\prime}\right) 1$ or $v=2 \phi\left(v^{\prime}\right) 0$. But then $0^{-1} v 2$ or $1 v 0^{-1}$ is an element of $\mathcal{X}$ not of the form $00 u^{\prime} 00$, a contradiction. Hence $n_{0}=\max _{0}(n)$. If $\operatorname{MJ}_{0}(n)=0$, then $\max _{0}(n-1)=n_{0}$ and there is a factor $v$ of odd length $n-1$ with even number $n_{0}$ of 0 's. We must have $v=2 \phi\left(v^{\prime}\right)$ or $v=\phi\left(v^{\prime}\right) 1$ but then $1 v$ or $v 2$ is an element of $\mathcal{X}$ not of the form $00 u^{\prime} 00$, a contradiction and $\mathrm{MJ}_{0}(n)=1$.

For the other direction, assume that $n_{0}=\max _{0}(n)$ and $\mathrm{MJ}_{0}(n)=1$. In particular, $\max _{0}(n-1)=n_{0}-1$. Assume there exists a factor $u$ of $\mathcal{X}$ not of the form $u=00 u^{\prime} 00$. Since $u$ has even length and even number of 0's, we must have $u=01 u^{\prime} 20$ or $u$ has its first or last letter $y$ not equal to 0 . In the first case, $v=001 u^{\prime}$ has length $n-1$ and $n_{0}$ zeros, a contradiction. In the second case, removing the letter $y$ leads also to a factor of length $n-1$ with $n_{0}$ zeros.

The second part of the lemma is similar. Assume first that all the elements of $\mathcal{X}$ are preceded and followed by 00 . In particular, $n_{0}$ is even. If $n_{0} \neq \min _{0}(n)$, there is a factor $v$ of length $n$ with $n_{0}-1$ zeros. Since $|v|_{0}$ is odd but $n$ is even, we must have $v=0 \phi\left(v^{\prime}\right) 1$ or $v=2 \phi\left(v^{\prime}\right) 0$ but then $0 v 1^{-1}$ or $2^{-1} v 0$ is an element of $\mathcal{X}$ that starts or ends with 00 
and so is preceded or followed by 12 , a contradiction. Hence we have $n_{0}=\min _{0}(n)$. If $\operatorname{mj}_{0}(n)=0$, then $\min _{0}(n+1)=n_{0}$ and there is a factor $v$ of odd length $n+1$ with even number $n_{0}$ of 0 's. We must have $v=2 \phi\left(v^{\prime}\right)$ or $v=\phi\left(v^{\prime}\right) 1$ but then $\phi\left(v^{\prime}\right)$ is an element of $\mathcal{X}$ without a 00 preceding or following it.

For the other direction, assume that $n_{0}=\min _{0}(n)$ and $\operatorname{mj}_{0}(n)=1$. In particular $\min _{0}(n+1)=n_{0}+1$. If there exists a factor $u$ of $\mathcal{X}$ such that $1 u, 2 u, u 1$ or $u 2$ is a factor, then $\min _{0}(n+1) \leqslant n_{0}$, a contradiction. Hence all the factors $u$ of $\mathcal{X}$ can only be extended by $0 u 0$. Finally, note that $u \in \mathcal{X}$ cannot occur in $\mathbf{x}$ at odd index. In other words, any $u \in \mathcal{X}$ can be de-substituted. Indeed, if it is not the case, then $u$ is of the form $0 \phi\left(u^{\prime}\right) 0$, $0 \phi\left(u^{\prime}\right) 1,2 \phi\left(u^{\prime}\right) 0$ or $2 \phi\left(u^{\prime}\right) 1$. If $u$ is of the first form, then $\phi\left(u^{\prime}\right) 001$ is a factor of length $n+1$ with only $n_{0}$ zeros, which is a contradiction. Otherwise, $u$ is of one of the last three forms. Then either $u 2$ or $1 u$ is a factor of $\mathbf{x}$, which is not possible. So the only extension of $u$ as a factor of $\mathbf{x}$ is $00 u 00$.

Lemma 40. Let $n$ be a positive even integer and $n_{0}$ even such that $\min _{0}(n) \leqslant n_{0} \leqslant$ $\max _{0}(n)$. Let $\mathcal{X}$ be an abelian equivalence class of factors of $\mathbf{x}$ of length $n$ with $n_{0}$ zeros. The class $\mathcal{X}$ leads to only one 2-abelian equivalence class of $\mathbf{p}$ if and only if $n_{0}=\min _{0}(n)$ and $\operatorname{mj}_{0}(n)=1$ or $n_{0}=\max _{0}(n)$ and $\mathrm{MJ}_{0}(n)=1$. Otherwise, $\mathcal{X}$ splits into two classes.

Proof. The factors in $\mathbf{x}$ of length $n=2$ are $00,01,12,21,20$. The two classes to consider are $\mathcal{X}_{1}=\{00\}$, which leads to one class, and $\mathcal{X}_{2}=\{12,21\}$, which splits into two classes. Since $\mathrm{MJ}_{0}(2)=1$ and $\mathrm{mj}_{0}(2)=0$, the proposition is true.

Hence let $n \geqslant 4$ even. If $n_{0}=\min _{0}(n)$ and $\operatorname{mj}_{0}(n)=1$, then by Lemma 39, all the elements of $\mathcal{X}$ are preceded by 00 . In particular, they all start with 1 and $\mathcal{X}$ leads to only one 2-abelian equivalence class. Similarly, if $n_{0}=\max _{0}(n)$ and $\mathrm{MJ}_{0}(n)=1$, then by Lemma 39, all the elements of $\mathcal{X}$ start with 0 and we have only one class.

Assume now that $\mathcal{X}$ leads to only one class. If an element $u$ of $\mathcal{X}$ starts with 2 , we have $u=2 \phi\left(u^{\prime}\right) 1$ since $n$ and $n_{0}$ are even. Then $1 u 1^{-1}$ is an element of $\mathcal{X}$ starting with 1 and $\mathcal{X}$ splits into two classes. Hence every element $u$ of $\mathcal{X}$ starts with 0 or 1 . Assume there exists a factor $u$ in $\mathcal{X}$ that starts with a 1 . Then $u=12 \phi\left(u^{\prime}\right)$ and $u$ cannot be followed by a 1 since otherwise $1^{-1} u 1$ would be an element of $\mathcal{X}$ starting with 2 . Hence $u$ is always followed by 00 and so ends with 12 . Similarly, it can only be preceded by 00 . Hence all the factors in $\mathcal{X}$ starting with a 1 are preceded and followed by 00 . In particular, if a factor in $\mathcal{X}$ starts with 1 and occurs in $\mathbf{x}$ at index $i$, then the two factors starting at indices $i-1$ and $i+1$ in $\mathbf{x}$ have $n_{0}+1$ zeros. Assume now there exists a factor $u$ in $\mathcal{X}$ starting with a 0 . Then, $u$ can be de-substituted. Otherwise, as $n$ and $n_{0}$ are even, $u$ is of the form $0 \phi\left(u^{\prime}\right) 0$ where $\phi\left(u^{\prime}\right)$ ends with 12. Thus $2 \phi\left(u^{\prime}\right) 2^{-1}$ is an element of $\mathcal{X}$ starting with 2 , which is a contradiction. Hence $u$ starts with 00 . If $u$ ends with 12 , then again, $2 u 2^{-1}$ is an element of $\mathcal{X}$ starting with 2 . Hence $u=00 \phi\left(u^{\prime}\right) 00$ and all elements of $\mathcal{X}$ starting with 0 start and end with 00 . In particular, if a factor in $\mathcal{X}$ starts with 0 and occurs in $\mathbf{x}$ at index $i$, then the two factors starting at indices $i-1$ and $i+1$ in $\mathbf{x}$ have $n_{0}-1$ zeros.

If no elements of $\mathcal{X}$ start with 1 or no elements start with 0, we are done by Lemma 39. 
Otherwise, since one can show that $\mathbf{x}$ is uniformly recurrent ${ }^{3}$, we can assume that there exist a factor $u \in \mathcal{X}$ that starts with 0 and occurs at index $i$ in $\mathbf{x}$, and a factor $v \in \mathcal{X}$ that starts with 1 and occurs at index $i+\ell$ in $\mathbf{x}$, such that any factor $w_{s}$ of length $n$ occurring at index $i+s$ in $\mathbf{x}$ does not belong to $\mathcal{X}$ for $0<s<\ell$. Then $w_{1}$ has $n_{0}-1$ zeros whereas $w_{\ell-1}$ has $n_{0}+1$ zeros. But there is no factor $w_{s}$ with $n_{0}$ zeros. This is a contradiction since the number of 0's changes by at most one between two factors of the same length starting at consecutive indexes.

Proof of Proposition 34. The case $n$ odd is given by Corollary 37. Assume now that $n$ is even. Then by Lemma $19, \min _{0}(n)$ and $\max _{0}(n)$ are even, and therefore $\Delta_{0}(n)$ is even as well. Let $\mathcal{X}$ be an abelian equivalence class of factors of $\mathbf{x}$ of length $n$. Let $n_{0}$ be the number of 0 's in the elements of $\mathcal{X}$. There are exactly $\frac{\Delta_{0}(n)}{2}$ odd values of $n_{0}$ and $\frac{\Delta_{0}(n)}{2}+1$ even values. By Corollary 38 , if $n_{0}$ is odd, $\mathcal{X}$ leads to one 2-abelian equivalence class of p. By Lemma $40, \mathcal{X}$ splits into two classes except for $n_{0}=\min _{0}(n)$ if $\operatorname{mj}_{0}(n)=1$ and for $n_{0}=\max _{0}(n)$ if $\operatorname{MJ}_{0}(n)=1$. Hence there are in total $\frac{\Delta_{0}(n)}{2}+1-\mathrm{MJ}_{0}(n)-\operatorname{mj}_{0}(n)$ cases where $\mathcal{X}$ leads to two 2-abelian equivalence classes of $\mathbf{p}$ instead of one and this is exactly the difference between $\mathcal{P}_{\mathbf{p}}^{(2)}(n+1)$ and $\mathcal{P}_{\mathbf{x}}^{(1)}(n)$.

Corollary 41. The sequence $\mathcal{P}_{\mathbf{p}}^{(2)}(n)_{n \geqslant 0}$ is 2-regular.

Proof. We can make use of Lemma 8. Thanks to Proposition $34, \mathcal{P}_{\mathbf{p}}^{(2)}(n+1)$ can be expressed as a combination of $\mathcal{P}_{\mathbf{x}}^{(1)}(n), \Delta_{0}(n), \mathrm{MJ}_{0}(n), \mathrm{mj}_{0}(n)$ using the predicate $(n$ mod $2)$. Note that the predicate $(n \bmod 2)$ is trivially 2 -automatic.

We proved the 2-regularity of $\mathcal{P}_{\mathbf{x}}^{(1)}(n)_{n \geqslant 0}$ and of $\Delta_{0}(n)_{n \geqslant 0}$ in Section 4 . Observe that

$$
\operatorname{MJ}_{0}(n+1)=\max _{0}(n+1)-\max _{0}(n)=\min _{0}(n+1)+\Delta_{0}(n+1)-\min _{0}(n)-\Delta_{0}(n) .
$$

Since $\mathrm{MJ}_{0}(n+1)$ can only take the values 0 and 1 , the latter relation can also be expressed using $\left(\min _{0}(n) \bmod 2\right)_{n \geqslant 0}$ and $\left(\Delta_{0}(n) \bmod 2\right)_{n \geqslant 0}$. These latter sequences are 2-regular by Corollary 26. By Lemma 11, $\mathrm{MJ}_{0}(n+1)_{n \geqslant 0}$ is thus a combination of four 2-regular sequences. Applying again Lemma $11, \mathrm{MJ}_{0}(n)_{n \geqslant 0}$ is also 2-regular. We can show similarly that $\mathrm{mj}_{0}(n)_{n \geqslant 0}$ is 2-regular. In fact, both sequences $\mathrm{MJ}_{0}(n)_{n \geqslant 0}$ and $\mathrm{mj}_{0}(n)_{n \geqslant 0}$ are 2automatic since they only take values 0 and 1 . Thus, all the functions in the expression for $\mathcal{P}_{\mathbf{p}}^{(2)}(n+1)$ are 2-regular.

Finally, as $\mathcal{P}_{\mathbf{p}}^{(2)}(n+1)_{n \geqslant 0}$ is 2-regular, $\mathcal{P}_{\mathbf{p}}^{(2)}(n)_{n \geqslant 0}$ is 2-regular by Lemma 11 .

\section{Abelian complexity of block(t,2)}

In this section, we turn our attention to the Thue-Morse word $\mathbf{t}$. Let $\mathbf{y}$ denote

$$
\operatorname{block}(\mathbf{t}, 2)=132120132012132120121320 \cdots,
$$

\footnotetext{
${ }^{3} \mathrm{~A}$ word is uniformly recurrent if every factor occurs infinitely often and, for each factor, there is a constant $c$ such that two consecutive occurrences of the factor occur within $c$ of each other. To prove that $\mathbf{x}$ is uniformly recurrent, it is enough to observe that $\phi$ is primitive since for each letter $y \in\{0,1,2\}$, $\phi^{3}(y)$ contains all the letters.
} 
the 2-block coding of $\mathbf{t}$ introduced in Example 17. Recall that $\mathbf{y}$ is a fixed point of the morphism $\nu$ defined by $\nu: 0 \mapsto 12,1 \mapsto 13,2 \mapsto 20,3 \mapsto 21$. The approach here is similar to that of the period-doubling word: we consider in this section the abelian complexity of $\mathbf{y}$, and then we compare $\mathcal{P}_{\mathbf{y}}^{(1)}(n)$ with $\mathcal{P}_{\mathbf{t}}^{(2)}(n)$ in Section 7.

Our study of the period-doubling word in Sections 4 and 5 made substantial use of counting 0's in factors of $\mathbf{x}$. Alternatively, we could have counted the total number of 1 's and 2's in factors of $\mathbf{x}$, since this is equivalent information and since the letters 1 and 2 alternate in $\mathbf{x}$.

For the Thue-Morse word, the appropriate statistic for factors of $\mathbf{y}$ is the total number of 1's and 2's (or, equivalently, the total number of 0's and 3's). We will show in Lemma 45 that the letters 1 and 2 alternate in $\mathbf{y}$. Therefore, for $n \in \mathbb{N}$ we set

$$
\begin{aligned}
\max _{12}(n) & :=\max \left\{|u|_{1}+|u|_{2}: u \text { is a factor of } \mathbf{y} \text { with }|u|=n\right\} \\
\min _{12}(n) & :=\min \left\{|u|_{1}+|u|_{2}: u \text { is a factor of } \mathbf{y} \text { with }|u|=n\right\} \\
\Delta_{12}(n) & :=\max _{12}(n)-\min _{12}(n)
\end{aligned}
$$

Remark 42. Note that $g(\mathbf{y})$ is exactly the period-doubling word $\mathbf{p}$, where $g$ is the coding defined by $g(0)=1, g(1)=0, g(2)=0$ and $g(3)=1$. In particular, $\Delta_{12}(n)+1$ is the abelian complexity function of the period-doubling word. This function was also studied in $[5,14]$. Here we obtain relations of the same type as the relations in Theorem 4.

The fact that $\mathcal{P}_{\mathbf{y}}^{(1)}(n)_{n \geqslant 0}$ is 2-regular will follow from the next statement.

Proposition 43. Let $n \in \mathbb{N}$. We have

$$
\mathcal{P}_{\mathbf{y}}^{(1)}(n)= \begin{cases}2 \Delta_{12}(n)+2 & \text { if } n \text { is odd } \\ \frac{5}{2} \Delta_{12}(n)+\frac{5}{2} & \text { if } n \text { and } \Delta_{12}(n)+1 \text { are even } \\ \frac{5}{2} \Delta_{12}(n)+4 & \text { if } n, \Delta_{12}(n) \text { and } \min _{12}(n)+1 \text { are even } \\ \frac{5}{2} \Delta_{12}(n)+1 & \text { if } n, \Delta_{12}(n) \text { and } \min _{12}(n) \text { are even. }\end{cases}
$$

To be able to apply the composition result given by Lemma 8 to the expression of $\mathcal{P}_{\mathbf{y}}^{(1)}$ derived in Proposition 43, we have therefore to prove that

- the sequence $\Delta_{12}(n)_{n \geqslant 0}$ is 2-regular and

- the predicates occurring in (3) are 2-automatic.

Section 6.1 is dedicated to the proof of Proposition 43. In Section 6.2, we give a proof of the two previous items. In particular, we show that $\Delta_{12}(n)_{n \geqslant 0}$ satisfies a reflection symmetry. This permits us to express recurrence relations for $\mathcal{P}_{\mathbf{y}}^{(1)}$ at the end of Section 6.2. 


\subsection{Proof of Proposition 43}

We first need three technical lemmas about factors of $\mathbf{y}=\operatorname{block}(\mathbf{t}, 2)$.

Lemma 44. The set of factors of $\mathbf{y}$ of length 2 is $\mathrm{Fac}_{\mathbf{y}}(2)=\{01,12,13,20,21,32\}$.

Proof. It is easy to check that these six words are factors. To prove that they are the only ones, it is enough to check that for any element $u$ in $\{01,12,13,20,21,32\}$ the three factors of length 2 of $\nu(u)$ are still in $\{01,12,13,20,21,32\}$.

The following lemma has already been observed in [14, Lemma 10].

Lemma 45. If $w$ is a factor of $\mathbf{y}$, then $\left.|| w\right|_{1}-|w|_{2} \mid \leqslant 1$ and $\left.|| w\right|_{0}-|w|_{3} \mid \leqslant 1$. In particular, the letters 1 and 2 (respectively 0 and 3 ) alternate in $\mathbf{y}$.

Proof. First note that if for all factors of a word $u$, the numbers of two letters $x$ and $y$ differ by at most 1 , then $x$ and $y$ alternate in $u$. Furthermore, if the first or the last occurrence of one of these letters is $x$, then $|u|_{x} \geqslant|u|_{y}$. If both the first and the last occurrences are $x$, then $|u|_{x}=|u|_{y}+1$.

We prove the result by induction on the length $\ell$ of the factor. The result is true for factors of length $\ell=1$. Let $w$ be a factor of length $\ell>1$ and assume the result holds for factors of length smaller than $\ell$. If $w$ can be de-substituted as $w=\nu\left(w^{\prime}\right)$, we have

$$
\begin{aligned}
& |w|_{0}=\left|w^{\prime}\right|_{2}, \\
& |w|_{1}=\left|w^{\prime}\right|_{0}+\left|w^{\prime}\right|_{1}+\left|w^{\prime}\right|_{3}, \\
& |w|_{2}=\left|w^{\prime}\right|_{0}+\left|w^{\prime}\right|_{2}+\left|w^{\prime}\right|_{3}, \\
& |w|_{3}=\left|w^{\prime}\right|_{1} .
\end{aligned}
$$

Using the induction hypothesis, we have

$$
\left.|| w\right|_{1}-|w|_{2}|=||w|_{0}-|w|_{3}|=|\left|w^{\prime}\right|_{1}-\left|w^{\prime}\right|_{2} \mid \leqslant 1 .
$$

If $w$ cannot be de-substituted and has odd length, we have

$$
w \in\left\{1^{-1} \nu\left(w^{\prime}\right), 2^{-1} \nu\left(w^{\prime}\right), \nu\left(w^{\prime}\right) 1, \nu\left(w^{\prime}\right) 2\right\}
$$

for some factor $w^{\prime}$ with $\left|w^{\prime}\right|<\ell$. Assume that $w=1^{-1} \nu\left(w^{\prime}\right)$. Then as before $\left.|| w\right|_{0}-|w|_{3} \mid=$ $\left.|| w^{\prime}\right|_{1}-\left|w^{\prime}\right|_{2} \mid \leqslant 1$. For the numbers of 1 and $2, w^{\prime}$ starts with 0 or 1 . Since by Lemma 44 a 0 is always followed by a $1, w^{\prime}$ starts either with 01 or with 1 . In both cases, since 1 and 2 alternate, we have $\left|w^{\prime}\right|_{1} \geqslant\left|w^{\prime}\right|_{2}$ and thus

$$
\left.|| w\right|_{1}-|w|_{2}|=|\left|w^{\prime}\right|_{1}-\left|w^{\prime}\right|_{2}-1 \mid \leqslant 1 \text {. }
$$

The same reasoning can be done for $w=2^{-1} \nu\left(w^{\prime}\right)$. If $w=\nu\left(w^{\prime}\right) 1$, then we clearly have $\left.|| w\right|_{0}-|w|_{3} \mid \leqslant 1$ using the result on $\nu\left(w^{\prime}\right)$. By Lemma 44, the factor $\nu\left(w^{\prime}\right)$ must end 
either with 0 or 2 . So $w^{\prime}$ ends with 0 or 2 as well. Since a 0 is always preceded by a 2, we necessarily have $\left|w^{\prime}\right|_{2} \geqslant\left|w^{\prime}\right|_{1}$ and

$$
\left.|| w\right|_{1}-|w|_{2}|=|\left|w^{\prime}\right|_{1}-\left|w^{\prime}\right|_{2}+1 \mid \leqslant 1
$$

The same reasoning applies to $w=\nu\left(w^{\prime}\right) 2$.

If $w$ cannot be de-substituted and has even length, then we have

$$
w \in\left\{1^{-1} \nu\left(w^{\prime}\right) 1,1^{-1} \nu\left(w^{\prime}\right) 2,2^{-1} \nu\left(w^{\prime}\right) 1,2^{-1} \nu\left(w^{\prime}\right) 2\right\}
$$

for some factor $w^{\prime}$ with $\left|w^{\prime}\right|<\ell$. If the same letter is removed and added to $\nu\left(w^{\prime}\right)$, then the result is clearly true. Otherwise, assume that $w=1^{-1} \nu\left(w^{\prime}\right) 2$ (the same reasoning holds for the last case). It is clear that $\left.|| w\right|_{0}-|w|_{3} \mid \leqslant 1$ using the result on $\nu\left(w^{\prime}\right)$. For the numbers of 1 and 2, as before, $w^{\prime}$ starts with 01 or 1 and ends with 13 or 1 . Hence we have $\left|w^{\prime}\right|_{1}=\left|w^{\prime}\right|_{2}+1$ and then

$$
\left.|| w\right|_{1}-|w|_{2}|=|\left|w^{\prime}\right|_{1}-\left|w^{\prime}\right|_{2}-2 \mid \leqslant 1 \text {. }
$$

Lemma 46. Let $\tau, \tau^{\prime}$ be the morphisms respectively defined by

$$
\tau:\left\{\begin{array}{l}
0 \mapsto 0 \\
1 \mapsto 2 \\
2 \mapsto 1 \\
3 \mapsto 3
\end{array} \quad \text { and } \quad \tau^{\prime}:\left\{\begin{array}{l}
0 \mapsto 3 \\
1 \mapsto 1 \\
2 \mapsto 2 \\
3 \mapsto 0
\end{array} .\right.\right.
$$

If $w$ is a factor of $\mathbf{y}$, then $\tau^{\prime}(w)^{\mathrm{R}}, \tau(w)^{\mathrm{R}}$ and $\tau^{\prime}(\tau(w))$ are also factors of $\mathbf{y}$.

Proof. We prove the lemma for $\tau^{\prime}(w)^{\mathrm{R}}$ and $\tau(w)^{\mathrm{R}}$ since $\tau^{\prime}(\tau(w))=\tau^{\prime}\left(\tau(w)^{\mathrm{R}}\right)^{\mathrm{R}}$.

We first prove by induction that for any factor $u$ starting with the letter $x$ and ending with the letter $y$,

$$
\tau^{\prime}(\nu(u))^{\mathrm{R}}=a^{-1} \nu\left(\tau(u)^{\mathrm{R}}\right) b
$$

where $a=1$ (respectively $a=2, b=1, b=2$ ) if and only if $y \in\{0,2\}$ (resp. $y \in\{1,3\}$, $x \in\{0,1\}, x \in\{2,3\})$. Note that $a^{-1} \nu\left(\tau(u)^{\mathrm{R}}\right) b$ is well defined. Indeed, if $y \in\{0,2\}$, then $\tau(u)^{\mathrm{R}}$ starts with 0 or 1 and thus $\nu\left(\tau(u)^{\mathrm{R}}\right)$ starts with $a=1$. The same holds with $y \in\{1,3\}$.

The relation (4) is true for $u$ of length 1 . We have for example

$$
\tau^{\prime}(\nu(0))^{\mathrm{R}}=21=1^{-1} \nu(0) 1=1^{-1} \nu\left(\tau(0)^{\mathrm{R}}\right) 1
$$

and

$$
\tau^{\prime}(\nu(1))^{\mathrm{R}}=01=2^{-1} \nu(2) 1=2^{-1} \nu\left(\tau(1)^{\mathrm{R}}\right) 1 .
$$

Let $u=u^{\prime} y x$ be a factor with at least two letters $x$ and $y$. Assume the conclusion holds for words of length at most $|u|-1$. By the induction hypothesis, we have $\tau^{\prime}\left(\nu\left(u^{\prime} y\right)\right)^{\mathrm{R}}=$ $a^{-1} \nu\left(\tau\left(u^{\prime} y\right)^{\mathrm{R}}\right) b$ and $\tau^{\prime}(\nu(x))^{\mathrm{R}}=c^{-1} \nu\left(\tau(x)^{\mathrm{R}}\right) d$ with appropriate $a, b, c, d$. Since $y x$ is a factor, one can check using Lemma 44 that $a=d$. Indeed, if $y \in\{0,2\}$, then $x \in\{0,1\}$. 
So $a=1$ and $d=1$. Similarly, if $y \in\{1,3\}$, then $x \in\{2,3\}$. Hence, $a=2$ and $d=2$. Thus, we have

$$
\begin{aligned}
\tau^{\prime}(\nu(u))^{\mathrm{R}} & =\tau^{\prime}\left(\nu\left(u^{\prime} y x\right)^{\mathrm{R}}\right) \\
& =\tau^{\prime}(\nu(x))^{\mathrm{R}} \tau^{\prime}\left(\nu\left(u^{\prime} y\right)\right)^{\mathrm{R}} \\
& =c^{-1} \nu\left(\tau(x)^{\mathrm{R}}\right) d a^{-1} \nu\left(\tau\left(u^{\prime} y\right)^{\mathrm{R}}\right) b \\
& =c^{-1} \nu\left(\tau\left(u^{\prime} y x\right)^{\mathrm{R}}\right) b \\
& =c^{-1} \nu\left(\tau(u)^{\mathrm{R}}\right) b .
\end{aligned}
$$

We can similarly prove by induction that for any factor $u$ starting with the letter $x$ and ending with the letter $y$,

$$
\tau(\nu(u))^{\mathrm{R}}=a^{-1} \nu\left(\tau^{\prime}(u)^{\mathrm{R}}\right) b
$$

where $a=1$ (respectively $a=2, b=1, b=2$ ) if and only if $y \in\{1,3\}$ (resp. $y \in\{0,2\}$, $x \in\{2,3\}, x \in\{0,1\})$.

We now prove the lemma (for $\tau$ and $\tau^{\prime}$ together) by induction on the length of $w$. One can check by hand that the lemma is true for $w$ of length at most 4. Assume the lemma is true for any factor of length at most $n \geqslant 4$, and let $w$ be a factor of length $n+1$. There exist some factors $s, t$ and $v$ such that $s w t=\nu(v), 0 \leqslant|t| \leqslant 1$ and $1 \leqslant|s| \leqslant 2$. Then we have $|v| \leqslant \frac{n+4}{2} \leqslant n$. By the induction hypothesis, $\tau(v)^{\mathrm{R}}$ is a factor of $\mathbf{y}$. Hence $\nu\left(\tau(v)^{\mathrm{R}}\right)$ is also a factor of $\mathbf{y}$. Using the previous result, $\tau^{\prime}(\nu(v))^{\mathrm{R}}=a^{-1} \nu\left(\tau(v)^{\mathrm{R}}\right) b$ for some letters $a$ and $b$. But we also have $\tau^{\prime}(\nu(v))^{\mathrm{R}}=\tau^{\prime}(t)^{\mathrm{R}} \tau^{\prime}(w)^{\mathrm{R}} \tau^{\prime}(s)^{\mathrm{R}}$ and since $s$ has at least one letter, $\tau^{\prime}(w)^{\mathrm{R}}$ is a factor of $\nu\left(\tau(v)^{\mathrm{R}}\right)$. Hence it is a factor of $\mathbf{y}$. We do the same proof for $\tau(w)^{\mathrm{R}}$.

We are now ready to prove the relationship between $\mathcal{P}_{\mathbf{y}}^{(1)}(n)$ and $\Delta_{12}(n)$.

Proof of Proposition 43. Let $u$ be a factor of length $n$ of $\mathbf{y}$. Let $n_{12}=|u|_{1}+|u|_{2}$ and $n_{03}=|u|_{0}+|u|_{3}$.

Assume first that $n$ is odd. If $n_{12}$ is even, then there are the same number of 1 's and 2's in $u$ by Lemma 45. Since $n_{13}$ is odd, if $|u|_{0}=|u|_{3}+1$ (resp. $|u|_{3}=|u|_{0}+1$ ), then $\tau^{\prime}(u)^{\mathrm{R}}$ is a factor by Lemma 46 and $\left|\tau^{\prime}(u)^{\mathrm{R}}\right|_{3}=\left|\tau^{\prime}(u)^{\mathrm{R}}\right|_{0}+1\left(\operatorname{resp} .\left|\tau^{\prime}(u)^{\mathrm{R}}\right|_{0}=\left|\tau^{\prime}(u)^{\mathrm{R}}\right|_{3}+1\right)$. In either case, $\tau^{\prime}(u)^{\mathrm{R}}$ still has $n_{12}$ ones and twos. Hence there are exactly two abelian equivalence classes for fixed $n$ odd and $n_{12}$ even. We can do the same reasoning if $n_{12}$ is odd. Finally, there are $\Delta_{12}(n)+1$ possible values for $n_{12}$ and thus $2\left(\Delta_{12}(n)+1\right)$ abelian equivalence classes for a fixed odd $n$.

Assume now that $n$ is even. If both $n_{12}$ and $n_{03}$ are even, then $u$ necessarily has the same number of 1's as 2's and the same number of 0's as 3's, and thus there is only one abelian equivalence class. Hence assume that $n_{12}$ and $n_{03}$ are odd. We have $\left(|u|_{0}-|u|_{3},|u|_{1}-|u|_{2}\right) \in\{-1,1\}^{2}$. By Lemma 46, the four factors $u, \tau^{\prime}(u)^{\mathrm{R}}, \tau(u)^{\mathrm{R}}$ and $\tau^{\prime}(\tau(u))$ realize the four possibilities for $\left(|u|_{0}-|u|_{3},|u|_{1}-|u|_{2}\right)$. Hence if $n_{12}$ and $n_{03}$ are both odd, there are four abelian equivalence classes. 
Now, we just have to count pairs $\left(n, n_{12}\right)$ with $n$ and $n_{12}$ even. If $\Delta_{12}(n)$ is odd, there are exactly $\left(\Delta_{12}(n)+1\right) / 2$ such pairs. So there are

$$
1 \cdot\left(\Delta_{12}(n)+1\right) / 2+4 \cdot\left(\Delta_{12}(n)+1\right) / 2=\frac{5}{2}\left(\Delta_{12}(n)+1\right)
$$

abelian classes for this value of $n$. If $\Delta_{12}(n)$ is even and $\min _{12}(n)$ is odd, there are exactly $\Delta_{12}(n) / 2$ even values for $n_{12}$, and so there are

$$
1 \cdot \Delta_{12}(n) / 2+4 \cdot\left(\Delta_{12}(n) / 2+1\right)=\frac{5}{2} \Delta_{12}(n)+4
$$

abelian classes. Finally, if $\Delta_{12}(n)$ is even and $\min _{12}(n)$ is even, there are $\Delta_{12}(n) / 2+1$ even values for $n_{12}$, and so there are

$$
1 \cdot\left(\Delta_{12}(n) / 2+1\right)+4 \cdot \Delta_{12}(n) / 2=\frac{5}{2} \Delta_{12}(n)+1
$$

abelian classes.

\section{$6.2 \Delta_{12}(n)_{n \geqslant 0}$ is 2-regular, $\left(\min _{12}(n) \bmod 2\right)_{n \geqslant 0}$ is 2-automatic}

In this section, we prove the following result.

Proposition 47. Let $\ell \geqslant 1$ and $r$ such that $0 \leqslant r<2^{\ell}$. We have

$$
\Delta_{12}\left(2^{\ell}+r\right)= \begin{cases}\Delta_{12}(r)+1 & \text { if } r \leqslant 2^{\ell-1} \\ \Delta_{12}\left(2^{\ell+1}-r\right) & \text { if } r>2^{\ell-1}\end{cases}
$$

Moreover,

$$
\min _{12}\left(2^{\ell}+r\right) \equiv \begin{cases}\min _{12}(r)+\ell \quad(\bmod 2) & \text { if } r \leqslant 2^{\ell-1} \\ \min _{12}\left(2^{\ell+1}-r\right)+\Delta_{12}\left(2^{\ell+1}-r\right) \quad(\bmod 2) & \text { if } r>2^{\ell-1}\end{cases}
$$

Note that those latter relations have a form similar to (but slightly different from) the assumptions of Theorem 4. Before giving the proof, we prove a corollary. The 2-regularity of $\mathcal{P}_{\mathbf{y}}^{(1)}(n)_{n \geqslant 0}$ follows from Proposition 43 and Corollary 48.

Corollary 48. The following statements are true.

- The sequence $\Delta_{12}(n)_{n \geqslant 0}$ is 2-regular.

- The sequence $\left(\Delta_{12}(n) \bmod 2\right)_{n \geqslant 0}$ is 2-automatic.

- The sequence $\left(\min _{12}(n) \bmod 2\right)_{n \geqslant 0}$ is 2-automatic. 
Proof. The first assertion is a direct consequence of Proposition 47 and Theorem 4. The second assertion follows from Lemma 10.

To prove the last assertion, we prove by induction that, modulo 2 ,

$$
\min _{12}(16 n+i) \equiv \begin{cases}\min _{12}(4 n) & \text { if } i=0 \\ \min _{12}(4 n+1) & \text { if } i \in\{1,4,5\} \\ \min _{12}(4 n+1)+1 & \text { if } i \in\{2,3\} \\ \min _{12}(4 n+2) & \text { if } i \in\{6,8,9\} \\ \min _{12}(4 n+2)+1 & \text { if } i \in\{7,10\} \\ \min _{12}(4 n+3) & \text { if } i \in\{12,13,15\} \\ \min _{12}(4 n+3)+1 & \text { if } i \in\{11,14\}\end{cases}
$$

and

$$
\Delta_{12}(16 n+i) \equiv \begin{cases}\Delta_{12}(4 n) & \text { if } i=0 \\ \Delta_{12}(4 n+1) & \text { if } i \in\{1,2,4\} \\ \Delta_{12}(4 n+1)+1 & \text { if } i \in\{3,5\} \\ \Delta_{12}(4 n+2) & \text { if } i=8 \\ \Delta_{12}(4 n+2)+1 & \text { if } i \in\{6,7,9,10\} \\ \Delta_{12}(4 n+3) & \text { if } i \in\{12,14,15\} \\ \Delta_{12}(4 n+3)+1 & \text { if } i \in\{11,13\} .\end{cases}
$$

The relations are true for $n=0$. Let $n>0$ and assume they are true for $n^{\prime}<n$. We can write $n=2^{\ell}+r$ with $\ell \geqslant 0$ and $0 \leqslant r<2^{\ell}$. Let $i \in\{0, \ldots, 15\}$. We consider two cases.

Assume first that $r<2^{\ell-1}$. We have $16 n+i=2^{\ell+4}+16 r+i$ and $16 r+i<2^{\ell+3}$.

$$
\begin{aligned}
\min _{12}(16 n+i) & \equiv \min _{12}(16 r+i)+\ell+4 \\
& \equiv \min _{12}(4 r+j)+\delta+\ell+4 \\
& \equiv \min _{12}\left(2^{\ell+2}+4 r+j\right)+\delta \\
& \equiv \min _{12}(4 n+j)+\delta \quad(\bmod 2)
\end{aligned}
$$

for some $j \in\{0, \ldots, 3\}$ and $\delta \in\{0,1\}$ according to the relations. A similar reasoning holds for the $\Delta_{12}$ relations.

Assume now that $r \geqslant 2^{\ell-1}$ and $i \neq 0$. Setting $i^{\prime}=16-i$ and $n^{\prime}=2^{\ell+1}-r-1$, we obtain $16 n^{\prime}+i^{\prime}=2^{\ell+5}-16 r-i$. It follows that, by Proposition 47,

$$
\begin{aligned}
\min _{12}(16 n+i) & \equiv \min _{12}\left(2^{\ell+5}-16 r-i\right)+\Delta_{12}\left(2^{\ell+5}-16 r-i\right) \\
& \equiv \min _{12}\left(16 n^{\prime}+i^{\prime}\right)+\Delta_{12}\left(16 n^{\prime}+i^{\prime}\right) \\
& \equiv \min _{12}\left(4 n^{\prime}+k\right)+\delta+\Delta_{12}\left(4 n^{\prime}+k^{\prime}\right)+\delta^{\prime}
\end{aligned}
$$

(induction)

for some $k, k^{\prime} \in\{0, \ldots, 3\}$ and $\delta, \delta^{\prime} \in\{0,1\}$ according to the relations. Note that we have 
$k=k^{\prime}$, so

$$
\begin{aligned}
\min _{12}(16 n+i) & \equiv \min _{12}\left(4 n^{\prime}+k\right)+\delta+\Delta_{12}\left(4 n^{\prime}+k\right)+\delta^{\prime} \\
& \equiv \min _{12}\left(2^{\ell+3}-(4 r+4-k)\right)+\delta+\Delta_{12}\left(2^{\ell+3}-(4 r+4-k)\right)+\delta^{\prime} \\
& \equiv \min _{12}\left(2^{\ell+2}+(4 r+4-k)\right)+\delta+\delta^{\prime} \\
& \equiv \min _{12}(4 n+(4-k))+\delta+\delta^{\prime} \quad(\bmod 2)
\end{aligned}
$$

Table 1 gives the values of $i^{\prime}, k, \delta$ and $\delta^{\prime}$ for all the values of $i \neq 0$. Observe that the values of $4-k$ and $\left(\delta+\delta^{\prime} \bmod 2\right)$ are the values given in the relation for $i$. To conclude the proof, consider the case $i=0$. We have

$$
\begin{aligned}
\min _{12}(16 n) & \equiv \min _{12}\left(16\left(2^{\ell+1}-r\right)\right)+\Delta_{12}\left(16\left(2^{\ell+1}-r\right)\right) \\
& \equiv \min _{12}\left(4\left(2^{\ell+1}-r\right)\right)+\Delta_{12}\left(4\left(2^{\ell+1}-r\right)\right) \\
& \equiv \min _{12}(4 n) \quad(\bmod 2)
\end{aligned}
$$

A similar reasoning works for the $\Delta_{12}$ relations.

\begin{tabular}{|c||c|c|c|c|c|c|c|c|c|c|c|c|c|c|c|}
\hline$i$ & 1 & 2 & 3 & 4 & 5 & 6 & 7 & 8 & 9 & 10 & 11 & 12 & 13 & 14 & 15 \\
\hline$i^{\prime}$ & 15 & 14 & 13 & 12 & 11 & 10 & 9 & 8 & 7 & 6 & 5 & 4 & 3 & 2 & 1 \\
\hline$k$ & 3 & 3 & 3 & 3 & 3 & 2 & 2 & 2 & 2 & 2 & 1 & 1 & 1 & 1 & 1 \\
\hline$\delta$ & 0 & 1 & 0 & 0 & 1 & 1 & 0 & 0 & 1 & 0 & 0 & 0 & 1 & 1 & 0 \\
\hline$\delta^{\prime}$ & 0 & 0 & 1 & 0 & 1 & 1 & 1 & 0 & 1 & 1 & 1 & 0 & 1 & 0 & 0 \\
\hline
\end{tabular}

Table 1: The corresponding values of $i^{\prime}=16-i, k, \delta$ and $\delta^{\prime}$.

Proposition 47 is a direct consequence of Lemmas 49, 52 and 54 given in this section.

Lemma 49. Let $\ell \in \mathbb{N}, \ell \geqslant 1$. We have $\Delta_{12}\left(2^{\ell}\right)=1, \min _{12}\left(2^{\ell}\right) \equiv \ell(\bmod 2)$,

$$
\min _{12}\left(2^{\ell}\right)+\max _{12}\left(2^{\ell+1}\right)=2^{\ell+1} \text { and } \max _{12}\left(2^{\ell}\right)+\min _{12}\left(2^{\ell+1}\right)=2^{\ell+1} .
$$

Proof. Let $\ell \geqslant 1, A_{\ell}=\frac{2^{\ell+1}+(-1)^{\ell}}{3}$ and $B_{\ell}=\frac{2^{\ell+1}+2(-1)^{\ell+1}}{3}$. The sequences

$$
\left(A_{\ell}\right)_{\ell \geqslant 1}=(1,3,5,11,21, \ldots) \text { and }\left(B_{\ell}\right)_{\ell \geqslant 1}=(2,2,6,10,22, \ldots)
$$

are integer sequences and both satisfy the recurrence relation $X_{\ell+1}=2^{\ell+1}-X_{\ell}$. Moreover we have $A_{\ell}=B_{\ell}+1$ for even $\ell$ and $B_{\ell}=A_{\ell}+1$ for odd $\ell$. Note that $\left|\nu^{\ell}(1)\right|_{1}+\left|\nu^{\ell}(1)\right|_{2}=A_{\ell}$ and $\left|\nu^{\ell}(0)\right|_{1}+\left|\nu^{\ell}(0)\right|_{2}=B_{\ell}$.

We show by induction that

$$
\left\{|w|_{1}+|w|_{2}: w \text { factor of } \mathbf{y} \text { with }|w|=2^{\ell}\right\}=\left\{A_{\ell}, B_{\ell}\right\} .
$$

Note that this result will imply the lemma and that we already have $A_{\ell}$ and $B_{\ell}$ in the set. 
It is easy to check the result for $\ell=1$. Assume the result is true for $\ell \geqslant 1$. Let $w$ be a factor of $\mathbf{y}$ of length $2^{\ell+1}$. If $w$ can be de-substituted, then $w=\nu(u)$ and $|w|_{1}+|w|_{2}=2|u|_{0}+|u|_{1}+|u|_{2}+2|u|_{3}$ as in the proof of Lemma 45. Hence $|w|_{1}+|w|_{2}=$ $2|u|-\left(|u|_{1}+|u|_{2}\right)=2^{\ell+1}-\left(|u|_{1}+|u|_{2}\right)$. Using the recurrence relation for $A_{\ell}$ and $B_{\ell}$ and since $|u|_{1}+|u|_{2} \in\left\{A_{\ell}, B_{\ell}\right\}$, we have $|w|_{1}+|w|_{2} \in\left\{A_{\ell+1}, B_{\ell+1}\right\}$. If $w$ cannot be de-substituted, then we can write $w=a^{-1} \nu(u) b$ for some letters $a, b \in\{1,2\}$ and $|\nu(u)|=2^{\ell+1}$. So $|w|_{1}+$ $|w|_{2}=|\nu(u)|_{1}+|\nu(u)|_{2}$. Since we already proved that $|\nu(u)|_{1}+|\nu(u)|_{2} \in\left\{A_{\ell+1}, B_{\ell+1}\right\}$, we are done.

To prove the second assertion of the lemma, observe that $\min _{12}\left(2^{\ell}\right)=A_{\ell}$ if $\ell$ is odd and $\min _{12}\left(2^{\ell}\right)=B_{\ell}$ if $\ell$ is even. Furthermore, $A_{\ell}$ is always odd whereas $B_{\ell}$ is always even.

In order to prove Lemmas 52 and 54, we first need some technical results.

Lemma 50. Let $u$ be a factor of $\mathbf{y}$ of length $n$. We have $|u|_{1}+|u|_{2}=\max _{12}(n)$ if and only if $|\nu(u)|_{1}+|\nu(u)|_{2}=\min _{12}(2 n)$, and $|u|_{1}+|u|_{2}=\min _{12}(n)$ if and only if $|\nu(u)|_{1}+|\nu(u)|_{2}=\max _{12}(2 n)$.

Proof. Recall that $|\nu(u)|_{1}+|\nu(u)|_{2}=2 n-\left(|u|_{1}+|u|_{2}\right)$. Assume that $|u|_{1}+|u|_{2}=\max _{12}(n)$ and that $|\nu(u)|_{1}+|\nu(u)|_{2}=x>\min _{12}(2 n)$. Thus $x=2 n-\max _{12}(n)$. There exists a factor $w$ of length $2 n$ with $x-1$ ones and twos. We can assume that $w$ can be de-substituted. Otherwise, we can write $w$ as $w=a^{-1} \nu(v) b$ for some $a, b \in\{1,2\}$. Thus $\nu(v)$ has the same length as $w$ and the same number of 1's and 2's. So we can assume $w=\nu(v)$. Then $|v|_{1}+|v|_{2}=2 n-(x-1)=\max _{12}(n)+1$, a contradiction.

For the other direction, assume that $|u|_{1}+|u|_{2}=x<\max _{12}(n)$ and that $|\nu(u)|_{1}+$ $|\nu(u)|_{2}=\min _{12}(2 n)$. Thus $x=n-\min _{12}(n)$. As before, there exists a factor $v$ of length $n$ with $x+1$ ones and twos. Then $\nu(v)$ has $\min _{12}(n)-1$ ones and twos, a contradiction.

The second part of the lemma is similar.

Lemma 51. Let $n$ be an odd integer. Then we have

$$
\begin{aligned}
\min _{12}(n) & =\min _{12}(n+1)-1 \\
\max _{12}(n) & =\max _{12}(n-1)+1
\end{aligned}
$$

Proof. Let $u$ be a factor of even length $n+1$ minimizing the number of 1's and 2's. Then either $u$ starts with 1 or 2 , or ends with 1 or 2 . Indeed, if $u$ can be de-substituted, then it starts with 1 or 2 . Otherwise, its last letter is the beginning of an image of $\nu$ and thus is 1 or 2 . Removing this letter, we get a word of length $n$ with $\min _{12}(n+1)-1$ ones and twos. Since the function $\min _{12}$ increases by 0 or 1 from $n$ to $n+1$, we have $\min _{12}(n)=\min _{12}(n+1)-1$.

For the second equality, consider a factor $u$ of even length $n-1$ with $\max _{12}(n-1)$ ones and twos. There exist two letters $a$ and $b$ such that $a u b$ is a factor. Then, as before, since $a u b$ has even length, $a$ or $b$ must be a 1 or a 2 . Then $a u$ or $u b$ is a factor of length $n$ with $\max _{12}(n-1)+1$ ones and twos and we conclude as before. 
Lemma 52. If $\ell \geqslant 1$ and $0 \leqslant r \leqslant 2^{\ell-1}$, then

$$
\begin{aligned}
\max _{12}\left(2^{\ell}+r\right) & =\max _{12}\left(2^{\ell}\right)+\max _{12}(r) \\
\min _{12}\left(2^{\ell}+r\right) & =\min _{12}\left(2^{\ell}\right)+\min _{12}(r) .
\end{aligned}
$$

Proof. We prove the two results together by induction on $\ell$. One checks the case $\ell=1$. Let $\ell>1$ and assume the result is true for $\ell-1$. Let $r$ such that $0 \leqslant r \leqslant 2^{\ell-1}$.

Assume first that $r$ is even. By the induction hypothesis, there exists a factor $u$ of length $2^{\ell-1}+r / 2$ such that

$$
|u|_{1}+|u|_{2}=\min _{12}\left(2^{\ell-1}+r / 2\right)=\min _{12}\left(2^{\ell-1}\right)+\min _{12}(r / 2) .
$$

We can write $u=v w$ with $v$ of length $2^{\ell-1}$ and $w$ of length $r / 2$. Both the words $v$ and $w$ must minimize the number of 1's and 2's for their respective lengths. By Lemma 50, $\nu(u)=\nu(v) \nu(w)$ maximizes the number of 1's and 2's and so do $\nu(v)$ and $\nu(w)$. Thus, $\max _{12}\left(2^{\ell}+r\right)=|\nu(u)|_{1}+|\nu(u)|_{2}$ and

$$
\max _{12}\left(2^{\ell}+r\right)=|\nu(v)|_{1}+|\nu(v)|_{2}+|\nu(w)|_{1}+|\nu(w)|_{2}=\max _{12}\left(2^{\ell}\right)+\max _{12}(r) .
$$

A similar proof shows that $\min _{12}\left(2^{\ell}+r\right)=\min _{12}\left(2^{\ell}\right)+\min _{12}(r)$.

Assume now that $r$ is odd. We still have $0 \leqslant r-1<r+1 \leqslant 2^{\ell-1}$. Hence we can apply the previous result to obtain $\max _{12}\left(2^{\ell}+r-1\right)=\max _{12}\left(2^{\ell}\right)+\max _{12}(r-1)$. By Lemma 51,

$$
\begin{aligned}
\max _{12}\left(2^{\ell}+r\right) & =\max _{12}\left(2^{\ell}+r-1\right)+1 \\
& =\max _{12}\left(2^{\ell}\right)+\max _{12}(r-1)+1 \\
& =\max _{12}\left(2^{\ell}\right)+\max _{12}(r) .
\end{aligned}
$$

For the $\min _{12}$ equality, a similar argument holds (using the previous result for $r+$ $1)$.

Lemma 53. If $\ell \geqslant 1$ and $2^{\ell-1} \leqslant r \leqslant 2^{\ell}$, then

$$
\begin{aligned}
\max _{12}\left(2^{\ell+1}\right) & =\max _{12}\left(2^{\ell}+r\right)+\min _{12}\left(2^{\ell}-r\right) \\
\min _{12}\left(2^{\ell+1}\right) & =\min _{12}\left(2^{\ell}+r\right)+\max _{12}\left(2^{\ell}-r\right) .
\end{aligned}
$$

Moreover, there is a factor of length $2^{\ell+1}$ maximizing (resp. minimizing) the number of 1 's and 2 's such that the prefix of length $2^{\ell}+r$ also maximizes (resp. minimizes) the number of 1 's and 2 's.

Proof. We proceed by induction on $\ell$. The result is true for $\ell=1$ since the only non-trivial case is $r=1$. Then $\max _{12}(4)=\max _{12}(3)+\min _{12}(1)$ and $\min _{12}(4)=\min _{12}(3)+\max _{12}(1)$ and the factors 2120 and 0132 satisfy the claim.

Let $\ell>1$ and assume the result is true for $\ell-1$. Let $r$ such that $2^{\ell-1} \leqslant r \leqslant 2^{\ell}$. Assume first that $r$ is even. Then $2^{\ell-2} \leqslant r / 2 \leqslant 2^{\ell-1}$. By the induction hypothesis, there 
is a factor $u$ of length $2^{\ell}$ minimizing the number of 1 's and 2's such that the prefix $v$ of length $2^{\ell-1}+r / 2$ minimizes the number of 1's and 2's. Thus we can write $u=v w$ and $|v|_{1}+|v|_{2}=\min _{12}\left(2^{\ell-1}+r / 2\right)$ and necessarily $|w|_{1}+|w|_{2}=\max _{12}\left(2^{\ell-1}-r / 2\right)$. By Lemma 50, $\nu(u)$ and $\nu(v)$ maximize the number of 1's and 2's and $\nu(w)$ minimizes the number of 1's and 2's. So we can conclude the result. A similar proof shows the other relation. If $r$ is odd, then we still have $2^{\ell-1} \leqslant r-1 \leqslant 2^{\ell}$ since $\ell>1$. Thus we can use the previous result and together with Lemma 51 , we have

$$
\begin{aligned}
\max _{12}\left(2^{\ell+1}\right) & =\max _{12}\left(2^{\ell}+r-1\right)+\min _{12}\left(2^{\ell}-r+1\right) \\
& =\max _{12}\left(2^{\ell}+r\right)-1+\min _{12}\left(2^{\ell}-r\right)+1 \\
& =\max _{12}\left(2^{\ell}+r\right)+\min _{12}\left(2^{\ell}-r\right) .
\end{aligned}
$$

Similarly, using the fact that $r+1 \leqslant 2^{\ell}$,

$$
\begin{aligned}
\min _{12}\left(2^{\ell+1}\right) & =\min _{12}\left(2^{\ell}+r+1\right)+\max _{12}\left(2^{\ell}-r-1\right) \\
& =\min _{12}\left(2^{\ell}+r\right)+1+\max _{12}\left(2^{\ell}-r\right)-1 \\
& =\min _{12}\left(2^{\ell}+r\right)+\max _{12}\left(2^{\ell}-r\right) .
\end{aligned}
$$

For the construction of the factors, one can construct them using the factor $\nu(u)$ maximizing the number of 1's and 2's given for $r-1$ and the factor $\nu\left(u^{\prime}\right)$ minimizing the number of 1's and 2's given for $r+1$ in the previous construction. Since $r$ is odd, the letter between the prefix $\nu(v)$ of length $2^{\ell}+r-1$ and $2^{\ell}+r$ of $\nu(u)$ is 1 or 2 . Since the prefix of length $2^{\ell}+r-1$ of $\nu(u)$ maximizes the number of 1 's and 2 's, so does the prefix of length $2^{\ell}+r$ of $\nu(u)$. For $\min _{12}$, consider $\nu\left(u^{\prime}\right)$. There exist letters $a$ and $b$ such that $w=a^{-1} \nu\left(u^{\prime}\right) b$ is still a factor. We must have $a, b \in\{1,2\}$. Then the prefix of length $2^{\ell}+r$ of $w$ minimizes the number of 1's and 2's.

The previous lemma permits us to reformulate some relations between the two sequences $\max _{12}(n)_{n \geqslant 0}$ and $\min _{12}(n)_{n \geqslant 0}$.

Lemma 54. If $\ell \geqslant 1$ and $2^{\ell-1} \leqslant r \leqslant 2^{\ell}$, then

$$
\begin{aligned}
\max _{12}\left(2^{\ell}+r\right) & =2^{\ell+1}-\min _{12}\left(2^{\ell+1}-r\right) \\
\min _{12}\left(2^{\ell}+r\right) & =2^{\ell+1}-\max _{12}\left(2^{\ell+1}-r\right) .
\end{aligned}
$$

Proof. From the previous lemma, we have

$$
\max _{12}\left(2^{\ell}+r\right)=\max _{12}\left(2^{\ell+1}\right)-\min _{12}\left(2^{\ell}-r\right) .
$$

By Lemma 49, we have $\max _{12}\left(2^{\ell+1}\right)=2^{\ell+1}-\min _{12}\left(2^{\ell}\right)$. Moreover, by Lemma 52 , since $0 \leqslant 2^{\ell}-r \leqslant 2^{\ell-1}$, we get

$$
\min _{12}\left(2^{\ell}-r\right)=\min _{12}\left(2^{\ell}+2^{\ell}-r\right)-\min _{12}\left(2^{\ell}\right) .
$$

Similar relations hold when changing $\max _{12}$ to $\min _{12}$. 
The proof of Proposition 47 about the reflection relation satisfied by $\Delta_{12}(n)$ and the recurrence relation of $\min _{12}(n)$ is now immediate.

Proof of Proposition 47. If $\ell \geqslant 1$ and $0 \leqslant r \leqslant 2^{\ell-1}$, then subtracting the two relations provided by Lemma 52 gives

$$
\Delta_{12}\left(2^{\ell}+r\right)=\Delta_{12}(\ell)+\Delta_{12}(r)
$$

and we can conclude using the first relation given in Lemma $49, \Delta_{12}\left(2^{\ell}\right)=1$. By Lemma 52, $\min _{12}\left(2^{\ell}+r\right) \equiv \min _{12}\left(2^{\ell}\right)+\min _{12}(r)(\bmod 2)$. The expression for $\min _{12}\left(2^{\ell}+r\right)$ follows since $\min _{12}\left(2^{\ell}\right) \equiv \ell(\bmod 2)$ by Lemma 49 .

If $\ell \geqslant 1$ and $2^{\ell-1}<r<2^{\ell}$, then subtracting the two relations provided by Lemma 54 permits us to conclude the proof of the expression claimed for $\Delta_{12}\left(2^{\ell}+r\right)$. Moreover, using Lemma 54, we get

$$
\begin{aligned}
\min _{12}\left(2^{\ell}+r\right) & \equiv \max _{12}\left(2^{\ell+1}-r\right) \quad(\bmod 2) \\
& \equiv \min _{12}\left(2^{\ell+1}-r\right)+\Delta_{12}\left(2^{\ell+1}-r\right) \quad(\bmod 2)
\end{aligned}
$$

Using Propositions 43 and 47, we can express recurrence relations for $\mathcal{P}_{\mathbf{y}}^{(1)}$ as we did for the proof of Theorem 21.

Theorem 55. Let $\ell \geqslant 2$ and $r$ such that $0 \leqslant r<2^{\ell}$. For $r \leqslant 2^{\ell-1}$, we have

$$
\mathcal{P}_{\mathbf{y}}^{(1)}\left(2^{\ell}+r\right)=\left\{\begin{array}{cc}
\mathcal{P}_{\mathbf{y}}^{(1)}(r)+2 & \text { if } r \text { is odd } \\
\mathcal{P}_{\mathbf{y}}^{(1)}(r)+1 & \text { if }\left(r, \Delta_{12}\left(2^{\ell}+r\right) \text { and } \min _{12}\left(2^{\ell}+r\right) \text { are even }\right) \\
& \text { or }\left(r \text { and } \Delta_{12}\left(2^{\ell}+r\right)+1\right. \text { are even } \\
\left.\quad \text { and } \min _{12}\left(2^{\ell}+r\right) \equiv \ell+1 \quad(\bmod 2)\right) \\
\mathcal{P}_{\mathbf{y}}^{(1)}(r)+4 \quad \text { otherwise. }
\end{array}\right.
$$

For $r>2^{\ell-1}$, we have $\mathcal{P}_{\mathbf{y}}^{(1)}\left(2^{\ell}+r\right)=\mathcal{P}_{\mathbf{y}}^{(1)}\left(2^{\ell+1}-r\right)$.

\section{$7 \quad$ 2-abelian complexity of the Thue-Morse word}

The aim of this section is to express, in Theorem 56, $\mathcal{P}_{\mathbf{t}}^{(2)}(n+1)$ in terms of $\mathcal{P}_{\mathbf{y}}^{(1)}(n)$, $\Delta_{12}(n),\left(\min _{12}(n) \bmod 2\right)$ and two new functions $\operatorname{MJ}_{03}(n)$ and $\operatorname{mj}_{03}(n)$ that are defined analogously to $\mathrm{MJ}_{0}(n)$ and $\mathrm{mj}_{0}(n)$ of Section 5 . Let

$$
\begin{aligned}
& \max _{03}(n):=\max \left\{|u|_{0}+|u|_{3}: u \text { is a factor of } \mathbf{y} \text { with }|u|=n\right\}, \\
& \min _{03}(n):=\min \left\{|u|_{0}+|u|_{3}: u \text { is a factor of } \mathbf{y} \text { with }|u|=n\right\},
\end{aligned}
$$

and let

$$
\begin{aligned}
\operatorname{MJ}_{03}(n) & := \begin{cases}1 & \text { if } \max _{03}(n)>\max _{03}(n-1) \\
0 & \text { otherwise, }\end{cases} \\
\operatorname{mj}_{03}(n) & := \begin{cases}1 & \text { if } \min _{03}(n+1)>\min _{03}(n) \\
0 & \text { otherwise }\end{cases}
\end{aligned}
$$


Theorem 56. For $n$ odd, we have

$$
\begin{aligned}
\mathcal{P}_{\mathbf{t}}^{(2)}(n+1)-\mathcal{P}_{\mathbf{y}}^{(1)}(n)= \\
\begin{cases}\Delta_{12}(n)+2-2 \mathrm{MJ}_{03}(n)-2 \mathrm{mj}_{03}(n) & \text { if } \min _{12}(n) \text { and } \Delta_{12}(n) \text { are even } \\
\Delta_{12}(n)+1-2 \mathrm{MJ}_{03}(n) & \text { if } \min _{12}(n) \text { and } \Delta_{12}(n)+1 \text { are even } \\
\Delta_{12}(n)+1-2 \mathrm{mj}_{03}(n) & \text { if } \min _{12}(n) \text { and } \Delta_{12}(n) \text { are odd } \\
\Delta_{12}(n) & \text { if } \min _{12}(n)+1 \text { and } \Delta_{12}(n) \text { are even. } .\end{cases}
\end{aligned}
$$

For $n$ even, we have

$$
\mathcal{P}_{\mathbf{t}}^{(2)}(n+1)-\mathcal{P}_{\mathbf{y}}^{(1)}(n)= \begin{cases}\frac{1}{2} \Delta_{12}(n)+1 & \text { if } \min _{12}(n) \text { and } \Delta_{12}(n) \text { are even } \\ \frac{1}{2} \Delta_{12}(n) & \text { if } \min _{12}(n)+1 \text { and } \Delta_{12}(n) \text { are even } \\ \frac{1}{2} \Delta_{12}(n)+\frac{1}{2} & \text { if } \Delta_{12}(n) \text { is odd. }\end{cases}
$$

As in Section 5, we study when an abelian equivalence class of $\mathbf{y}=\operatorname{block}(\mathbf{t}, 2)$ splits into two 2-abelian equivalence classes of $\mathbf{t}$. We have similar propositions.

Proposition 57. Let $u$ and $v$ be factors of $\mathbf{t}$ of length $n$. Let $u^{\prime}$ and $v^{\prime}$ be the 2-block codings of $u$ and $v$. The factors $u$ and $v$ are 2-abelian equivalent if and only if $u^{\prime}$ and $v^{\prime}$ (of length $n-1$ ) are abelian equivalent and either $u^{\prime}$ and $v^{\prime}$ both have first letter in $\{0,1\}$ or both have first letter in $\{2,3\}$.

Let $\mathcal{X}$ be an abelian equivalence class of factors of $\mathbf{y}$ of length $n$. For a letter $a$, let $n_{a}$ denote the number of $a$ 's in each element of $\mathcal{X}$ and let $n_{12}=n_{1}+n_{2}, n_{03}=n_{0}+n_{3}$.

Lemma 58. If $n_{12}$ is odd, then $\mathcal{X}$ leads to a unique 2-abelian equivalence class of $\mathbf{t}$.

Proof. Assume that $n_{1}>n_{2}$ (the other case is similar). Then a word of $\mathcal{X}$ cannot start with 2 since the letters 1 and 2 alternate in $\mathbf{y}$ by Lemma 45. It cannot start with 3 neither since $n_{1}>n_{2}$ and a 3 is always followed by 2 by Lemma 44 . Hence it starts with 0 or 1 . Thus $\mathcal{X}$ leads to a unique 2 -abelian equivalence class.

Lemma 59. If $n$ and $n_{12}$ are even, then $\mathcal{X}$ splits into two 2-abelian equivalence classes of $\mathbf{t}$.

Proof. If $n$ and $n_{12}$ are even, then $n_{03}$ is also even and thus $n_{1}=n_{2}$ and $n_{0}=n_{3}$. Let $u$ be an element of $\mathcal{X}$. Then $u^{\prime}=\tau^{\prime}(\tau(u))$ is also an element of $\mathcal{X}$. Moreover, the first letter of $u$ is in $\{0,1\}$ if and only if the first letter of $u^{\prime}$ is in $\{2,3\}$. Hence $\mathcal{X}$ splits into two 2-abelian equivalence classes.

So the last and hardest case happens when $n$ is odd and $n_{12}$ is even, i.e., when $n$ and $n_{03}$ are odd. The $\mathrm{MJ}_{03}$ and $\mathrm{mj}_{03}$ functions permit us to handle this case.

Lemma 60. Let $n$ and $n_{03}$ are odd. Let $a \in\{0,3\}$ (resp. $b \in\{0,3\}$ ) be the letter in majority (resp. in minority) in factors in $\mathcal{X}$, among $\{0,3\}$. 
- We have $n_{03}=\max _{03}(n)$ and $\operatorname{MJ}_{03}(n)=1$ if and only if every factor in $\mathcal{X}$ starts and ends with a.

- We have $n_{03}=\min _{03}(n)$ and $\operatorname{mj}_{03}(n)=1$ if and only if every factor in $\mathcal{X}$ is preceded and followed by $b$.

Proof. Assume that $a=0$ and $b=3$ (the other case is symmetric). We first prove the statement for the maximum. Assume that all the factors in $\mathcal{X}$ start and end with 0 . If $n_{03}<\max _{03}(n)$, by continuity of the number of 0 's and 3's and since $\mathbf{y}$ is uniformly recurrent, there exists a factor yuz such that the factor yu (resp. $u z$ ) is of length $n$ with $n_{03}$ (resp. $n_{03}+1$ ) zeros and threes. We necessarily have $z \in\{0,3\}$ and $u$ is not finishing with a letter in $\{0,3\}$. Since $y u$ has $n_{03}$ zeros and threes, $y u$ or $\tau^{\prime}(y u)^{\mathrm{R}}$ is an element of $\mathcal{X}$ that is either not finishing or not starting with 0 , a contradiction. Hence we have $n_{03}=\max _{03}(n)$. Assume now that $\max _{03}(n-1)=n_{03}$. There exists a factor $u$ of even length $n-1$ with $n_{03}$ zeros. Without loss of generality, we can assume that $u$ has more 0 's than 3's (otherwise one can consider $\tau^{\prime}(u)^{\mathrm{R}}$ by Lemma 46). Since $u$ has even length, either $u$ occurs at an even index in $\mathbf{y}$ and is always followed by 1 or 2 , or $u$ occurs at an odd index in $\mathbf{y}$ and is always preceded by 1 or 2 . In other words, there is a factor of the form $y u$ or $u y$ with $y \in\{1,2\}$. Then $y u$ or $u y$ is an element of $\mathcal{X}$ with the first or last letter different from 0 , a contradiction.

For the other direction, assume that $n_{03}=\max _{03}(n)$ and $\mathrm{MJ}_{03}(n)=1$. Let $u$ be a factor in $\mathcal{X}$. If $u=x u^{\prime}$ or $u=u^{\prime} x$ with $x \neq 0$, then $u^{\prime}$ has length $n-1$ and $n_{03}$ zeros and threes. Thus $\operatorname{MJ}_{03}(n)=0$, a contradiction.

The second statement is proved in the same way. Assume that all the factors in $\mathcal{X}$ are preceded and followed by 3 . If $n_{03}>\min _{03}(n)$, by continuity of the number of 0 's and 3 's and since $\mathbf{y}$ is uniformly recurrent, there exists a factor yuz such that the factor yu (resp. $u z$ ) is of length $n$ with $n_{03}$ (resp. $n_{03}-1$ ) zeros and threes. We necessarily have $z \in\{1,2\}$. Then as before $y u$ or $\tau^{\prime}(y u)^{\mathrm{R}}$ is and element of $\mathcal{X}$ that is either not always followed or not always preceded by 3 , a contradiction. Hence we have $n_{03}=\min _{03}(n)$. Assume now that $\min _{03}(n+1)=n_{03}$. There exists a factor $u$ of even length $n+1$ with $n_{03}$ zeros. Without loss of generality, we can assume that $u$ has more 0's than 3's (otherwise one can consider $\tau^{\prime}(u)^{\mathrm{R}}$ by Lemma 46). Since $u$ has even length, either $u$ occurs at an even index and starts with 1 or 2 or $u$ occurs at an odd index and ends with 1 or 2 . In other words, $u=y u^{\prime}$ or $u=u^{\prime} y$ with $y \in\{1,2\}$ and $u^{\prime}$ is an element of $\mathcal{X}$ preceded or followed by a letter different from 3 , a contradiction.

For the other direction, assume that $n_{03}=\min _{03}(n)$ and $\operatorname{mj}_{03}(n)=1$. Let $u$ be a factor in $\mathcal{X}$. If $u^{\prime}=u x$ or $u^{\prime}=x u$ is a factor with $x \in\{1,2\}$, then $u^{\prime}$ has length $n+1$ and $n_{03}$ zeros and threes. So $\operatorname{mj}_{03}(n)=0$, which is a contradiction. Observe also that it is impossible to have $0 u$ or $u 0$ as factors of $\mathbf{y}$ since $|u|_{0}>|u|_{3}$ by assumption and the letters 0 and 3 alternate in $\mathbf{y}$ by Lemma 45. The conclusion is immediate.

Lemma 61. If $n$ is odd and $n_{12}$ is even, then $\mathcal{X}$ leads to only one 2-abelian equivalence class of $\mathbf{t}$ if and only if $n_{03}=\min _{03}(n)$ and $\operatorname{mj}_{03}(n)=1$, or $n_{03}=\max _{03}(n)$ and $\mathrm{MJ}_{03}(n)=$ 1. Otherwise, $\mathcal{X}$ splits into two classes. 
Proof. If $n$ is odd and $n_{12}$ is even, then $n_{03}$ is even. Assume that $n_{0}>n_{3}$ (the other case is symmetric). If $n_{03}=\min _{03}(n)$ and $\operatorname{mj}_{03}(n)=1$ then, by Lemma 60 , all the factors in $\mathcal{X}$ start with 0 , and so $\mathcal{X}$ leads to only one class. If $n_{03}=\max _{03}(n)$ and $\operatorname{MJ}_{03}(n)=1$, then all the factors in $\mathcal{X}$ are preceded and followed by 3 . In particular, they all start with 2 and again $\mathcal{X}$ leads to only one class.

For the other direction, suppose that $\mathcal{X}$ leads to only one class. All the factors in $\mathcal{X}$ must start either with a letter in $\{0,1\}$ or with a letter in $\{2,3\}$. Assume first that all the elements of $\mathcal{X}$ start with 0 or 1 . Let $u$ be a factor in $\mathcal{X}$. If the first letter of $u$ is 1 , it must start with 120 since $u$ has more 0's than 3's. Thus $u$ is always preceded by 2 . It cannot end with 1 ( since $n_{1}=n_{2}$ ). So it must end with 0 or 2 . If $u=120 u^{\prime} 2$, then $2120 u^{\prime}$ is an element of $\mathcal{X}$ starting with 2 , which is a contradiction. If $u=120 u^{\prime} 0$ then $u 1$ is a factor of $\mathbf{y}$. So $20 u^{\prime} 01$ is an element of $\mathcal{X}$ starting with 2 , a contradiction. Hence $u$ cannot start with 1 and thus starts with 0 . Observe that, if $u$ does not end with 0 , then $\tau(u)^{\mathrm{R}}$ is still an element of $\mathcal{X}$ by Lemma 46 and $\tau(u)^{\mathrm{R}}$ does not start with 0 , a contradiction. Hence all the factors in $\mathcal{X}$ start and end with 0 . By Lemma 60 , we have $n_{03}=\max _{03}(n)$ and $\mathrm{MJ}_{03}(n)=1$.

Assume now that all the elements of $\mathcal{X}$ start with 2 or 3 . Since $n_{0}>n_{3}$, they all start with 2. Moreover, as $n_{1}=n_{2}$, they must end with 0 or 1 . If $u \in \mathcal{X}$ ends with 0 , then $\tau^{\prime}(u)^{\mathrm{R}} \in \mathcal{X}$ starts with 3 by Lemma 46, a contradiction. So all factors in $\mathcal{X}$ end with 1 . Let $u=2 u^{\prime} 1$ be an element of $\mathcal{X}$. By Lemma 44, the only possible extensions of $u$ as a factor of length $n+1$ of $\mathbf{y}$ are $1 u, 3 u, u 2$ and $u 3$. If $1 u$ is a factor of $\mathbf{y}$, then $12 u^{\prime} \in \mathcal{X}$ starts with 1 , which is a contradiction. If $u 2$ is factor of $\mathbf{y}$, then $\tau\left(u^{\prime} 12\right)^{\mathrm{R}} \in \mathcal{X}$ starts with 1 , a contradiction. Hence all the factors in $\mathcal{X}$ are preceded and followed by 3 in $\mathbf{y}$. By Lemma 60, this means that $n_{03}=\min _{03}(n)$ and $\operatorname{mj}_{03}(n)=1$.

We are now ready to prove Theorem 56 .

Proof of Theorem 56. The difference between $\mathcal{P}_{\mathbf{t}}^{(2)}(n+1)$ and $\mathcal{P}_{\mathbf{y}}^{(1)}(n)$ is the number of abelian equivalence classes of factors of length $n$ of $\mathbf{y}$ that split into two 2-abelian equivalence classes of factors of length $n+1$ of $\mathbf{t}$.

For even $n$, by Lemmas 58 and 59 , it happens when $n_{12}$ is even. The number of even values of $n_{12} \in\left\{\min _{12}(n), \ldots, \max _{12}(n)\right\}$ is

$$
\begin{cases}\frac{1}{2} \Delta_{12}(n)+1 & \text { if } \min _{12}(n) \text { and } \Delta_{12}(n) \text { are even } \\ \frac{1}{2} \Delta_{12}(n) & \text { if } \min _{12}(n)+1 \text { and } \Delta_{12}(n) \text { are even } \\ \frac{1}{2} \Delta_{12}(n)+\frac{1}{2} & \text { if } \Delta_{12}(n) \text { is odd, }\end{cases}
$$

which leads to the result.

For odd $n$, by Lemmas 58 and 61, it happens when $n_{12}$ is even, except if $n_{03}=\min _{03}(n)$ and $\operatorname{mj}_{03}(n)=1$ or $n_{03}=\max _{03}(n)$ and $\mathrm{MJ}_{03}(n)=1$. The number of such cases is

$$
\begin{cases}\frac{\Delta_{12}(n)}{2}+1-\mathrm{MJ}_{03}(n)-\operatorname{mj}_{03}(n) & \text { if } \min _{12}(n) \text { and } \Delta_{12}(n) \text { are even } \\ \frac{\Delta_{12}(n)+1}{2}-\mathrm{MJ}_{03}(n) & \text { if } \min _{12}(n) \text { and } \Delta_{12}(n)+1 \text { are even } \\ \frac{\Delta_{12}(n)+1}{2}-\operatorname{mj}_{03}(n) & \text { if } \min _{12}(n) \text { and } \Delta_{12}(n) \text { are odd } \\ \frac{\Delta_{12}(n)}{2} & \text { if } \min _{12}(n)+1 \text { and } \Delta_{12}(n) \text { are even. }\end{cases}
$$


Indeed, consider for example the case that $\min _{12}(n)$ and $\Delta_{12}(n)$ are even. First, there are $\frac{\Delta_{12}(n)}{2}+1$ even values of $n_{12}$. Second, since $\min _{12}(n)$ is even and $n$ is odd, we have $\max _{03}(n)=n-\min _{12}(n)$ odd. Since $\Delta_{12}(n)$ is even, $\max _{12}(n)$ is also even and $\min _{03}(n)$ is odd.

If $n$ is such that $\operatorname{mj}_{03}(n)=1\left(\operatorname{resp} . \mathrm{MJ}_{03}(n)=1\right)$ then the case $n_{03}=\min _{03}(n)$ and $\operatorname{mj}_{03}(n)=1\left(\right.$ resp. $n_{03}=\max _{03}(n)$ and $\left.\operatorname{MJ}_{03}(n)=1\right)$ indeed happens. So we have to remove 1, i.e., $\operatorname{mj}_{03}(n)$ or $\mathrm{MJ}_{03}(n)$ for each case.

As another example, consider the case that $\min _{12}(n)$ and $\Delta_{12}(n)$ are odd. Then $\max _{03}(n)$ is even and $\min _{03}(n)$ is odd. There are $\frac{\Delta_{12}(n)+1}{2}$ even values of $n_{12}$. We cannot have $n_{03}=\max _{03}(n)$ (for parity reasons) and thus we never have $n_{03}=\max _{03}(n)$ and $\mathrm{MJ}_{03}(n)=1$. But the case $n_{03}=\min _{03}(n)$ happens and thus we have to remove one case when $\operatorname{mj}_{03}(n)=1$.

Finally, observe that to each pair $\left(n, n_{12}\right)$, with $n$ odd and $n_{12}$ even, correspond two abelian equivalence classes of $\mathbf{y}$ (see the proof of Proposition 43). Each of these classes splits into two 2-abelian equivalence classes. Hence multiplying by 2 the number of pairs $\left(n, n_{12}\right)$, with $n$ odd and $n_{12}$ even, gives the result claimed for $n$ odd.

Corollary 62. The sequence $\mathcal{P}_{\mathbf{t}}^{(2)}(n)_{n \geqslant 0}$ is 2-regular.

Proof. We can make use of Lemma 8. Thanks to Theorem 56, $\mathcal{P}_{\mathbf{t}}^{(2)}(n+1)$ can be expressed as a combination of $\mathcal{P}_{\mathbf{y}}^{(1)}(n), \Delta_{12}(n), \mathrm{MJ}_{03}(n), \mathrm{mj}_{03}(n)$ using the predicates $(n \bmod 2)$, $\left(\Delta_{12}(n) \bmod 2\right)$ and $\left(\min _{12}(n) \bmod 2\right)$.

The sequences $\mathcal{P}_{\mathbf{y}}^{(1)}(n)_{n \geqslant 0}$ and $\Delta_{12}(n)_{n \geqslant 0}$ are 2-regular from Section 6 . Note that we have $\operatorname{MJ}_{03}(n+1)=\min _{12}(n)-\min _{12}(n+1)+1$ and

$$
\begin{aligned}
\operatorname{mj}_{03}(n) & =\max _{12}(n)-\max _{12}(n+1)+1 \\
& =\min _{12}(n)-\min _{12}(n+1)+\Delta_{12}(n)-\Delta_{12}(n+1)+1 .
\end{aligned}
$$

As $\operatorname{MJ}_{03}(n+1)$ and $\operatorname{mj}_{03}(n)$ can only take the values 0 and 1 , these relations can also be expressed using $\left(\min _{12}(n) \bmod 2\right)_{n \geqslant 0}$ and $\left(\Delta_{12}(n) \bmod 2\right)_{n \geqslant 0}$. Since these two latter sequences are 2-regular, the sequences $\left(\min _{12}(n+1) \bmod 2\right)_{n \geqslant 0}$ and $\left(\Delta_{12}(n+1) \bmod 2\right)_{n \geqslant 0}$ are 2-regular by Lemma 11 and so are $\operatorname{MJ}_{03}(n+1)_{n \geqslant 0}$ and $\mathrm{mj}_{03}(n)_{n \geqslant 0}$ by Lemma 8 . Thus, $\mathrm{MJ}_{03}(n)_{n \geqslant 0}$ is 2-regular by Lemma 11 .

Since all the functions (resp. all the predicates) occurring in the statement of Theorem 56 are 2-regular (resp. 2-automatic), the composition given in Lemma 8 implies that the sequence $\mathcal{P}_{\mathbf{t}}^{(2)}(n+1)_{n \geqslant 0}$ is 2-regular. Hence, by Lemma $11, \mathcal{P}_{\mathbf{t}}^{(2)}(n)_{n \geqslant 0}$ is 2-regular.

\section{Conclusions}

The two examples treated in this paper, namely the 2-abelian complexity of the perioddoubling word and the Thue-Morse word, suggest that a general framework to study the $\ell$-abelian complexity of $k$-automatic sequences may exist. As an example, we consider the 3-block coding of the period-doubling word,

$$
\mathbf{z}=\operatorname{block}(\mathbf{p}, 3)=240125252401240124 \cdots .
$$


The abelian complexity $\mathcal{P}_{\mathbf{z}}^{(1)}(n)_{n \geqslant 0}=(1,5,5,8,6,10,19,11, \ldots)$ seems to satisfy, for $\ell \geqslant 4$, the following relations (which are quite similar to what we have discussed so far)

$$
\mathcal{P}_{\mathbf{z}}^{(1)}\left(2^{\ell}+r\right)= \begin{cases}\mathcal{P}_{\mathbf{z}}^{(1)}(r)+5 & \text { if } r \leqslant 2^{\ell-1} \text { and } r \text { even } \\ \mathcal{P}_{\mathbf{z}}^{(1)}(r)+7 & \text { if } r \leqslant 2^{\ell-1} \text { and } r \text { odd } \\ \mathcal{P}_{\mathbf{z}}^{(1)}\left(2^{\ell+1}-r\right) & \text { if } r>2^{\ell-1}\end{cases}
$$

Then, the next step would be to relate $\mathcal{P}_{\mathbf{p}}^{(3)}$ with $\mathcal{P}_{\mathbf{z}}^{(1)}$ (and try to extend the developments from Section 5).

\section{Acknowledgments}

We thank Jeffrey Shallit for some motivating discussions we had about this problem at some early stage of development.

\section{References}

[1] Jean-Paul Allouche and Jeffrey Shallit, The ring of $k$-regular sequences, Theoretical Computer Science 98 (1992), 163-197.

[2] Jean-Paul Allouche and Jeffrey Shallit, The ring of $k$-regular sequences II, Theoretical Computer Science 307 (2003), 3-29.

[3] Jean-Paul Allouche and Jeffrey Shallit, Automatic sequences. Theory, applications, generalizations, Cambridge University Press, Cambridge, 2003.

[4] Jean Berstel and Christophe Reutenauer, Noncommutative rational series with applications, Encyclopedia of Mathematics and its Applications, 137, Cambridge University Press, Cambridge, 2011.

[5] Francine Blanchet-Sadri, James D. Currie, Narad Rampersad and Nathan Fox, Abelian complexity of fixed point of morphism $0 \mapsto 012,1 \mapsto 02,2 \mapsto 1$, INTEGERS 14 (2014) \#A11.

[6] Carpi, Arturo and D'Alonzo, Valerio, On factors of synchronized sequences, Theoretical Computer Science, 411 (2010), 3932-3937.

[7] Émilie Charlier, Narad Rampersad and Jeffrey Shallit, Enumeration and decidable properties of automatic sequences, Internat. J. Found. Comput. Sci. 23 (2012), no. 5, 1035-1066.

[8] Alan Cobham, Uniform tag sequences, Math. Systems Theory 6 (1972), 186-192.

[9] Samuel Eilenberg, Automata, Languages and Machines, Vol. A, Academic Press, New York, 1974.

[10] Anna Frid, On uniform DOL words, STACS'98, Lecture Notes in Computer Science, 1373 (1998), 544-554. 
[11] Florian Greinecker, On the 2-abelian complexity of Thue-Morse subwords, arXiv:1404.3906.

[12] Juhani Karhumäki, Generalized Parikh mappings and homomorphisms, Information and Control 47 (1980), 155-165.

[13] Juhani Karhumäki, Aleksi Saarela and Luca Q. Zamboni, On a generalization of Abelian equivalence and complexity of infinite words, J. Combin. Theory Ser. A 120 (2013), no. 8, 2189-2206.

[14] Juhani Karhumäki, Aleksi Saarela and Luca Q. Zamboni, Variations of the MorseHedlund theorem for $k$-abelian equivalence, DLT 2014, Lecture Notes in Computer Science 8633 (2014), 203-214.

[15] Blake Madill and Narad Rampersad, The abelian complexity of the paperfolding word, Discrete Math. 313 (2013), no. 7, 831-838.

[16] The OEIS Foundation, The On-Line Encyclopedia of Integer Sequences, http:// oeis.org.

[17] Michel Rigo and Élise Vandomme, 2-abelian complexity of the Thue-Morse sequence, http://hdl. handle.net/2268/135841, 2012. 